ARTICLE

\title{
MaTAR25 IncRNA regulates the Tensin1 gene to impact breast cancer progression
}

Kung-Chi Chang (1) 1,2, Sarah D. Diermeier (10) 1,8,9, Allen T. Yu 1,3,9, Lily D. Brine, Suzanne Russo1,

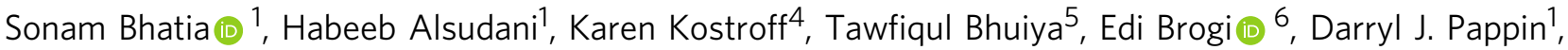
C. Frank Bennett ${ }^{7}$, Frank Rigo ${ }^{7}$ \& David L. Spector (1) ${ }^{1,2,3 凶}$

Misregulation of long non-coding RNA (IncRNA) genes has been linked to a wide variety of cancer types. Here we report on Mammary Tumor Associated RNA 25 (MaTAR25), a nuclear enriched and chromatin associated IncRNA that plays a role in mammary tumor cell proliferation, migration, and invasion, both in vitro and in vivo. MaTAR25 functions by interacting with purine rich element binding protein $B$ (PURB), and associating with a major downstream target gene Tensin1 (Tns1) to regulate its expression in trans. The Tns1 protein product is a critical component of focal adhesions linking signaling between the extracellular matrix and the actin cytoskeleton. Knockout of MaTAR25 results in down-regulation of Tns1 leading to a reorganization of the actin cytoskeleton, and a reduction of focal adhesions and microvilli. We identify LINC01271 as the human ortholog of MaTAR25, and importantly, increased expression of LINC01271 is associated with poor patient prognosis and metastasis. Our findings demonstrate that LINC01271 represents a potential therapeutic target to alter breast cancer progression.

\footnotetext{
${ }^{1}$ Cold Spring Harbor Laboratory, Cold Spring Harbor, NY 11724 New York, USA. ${ }^{2}$ Molecular and Cellular Biology Program, Stony Brook University, Stony Brook, NY 11794, USA. ${ }^{3}$ Genetics Program, Stony Brook University, Stony Brook, NY 11794, USA. ${ }^{4}$ Department of Surgical Oncology, Northwell Health, Lake Success, NY 11042, USA. ${ }^{5}$ Department of Pathology, Northwell Health, Lake Success, NY 11042, USA. ${ }^{6}$ Memorial Sloan Kettering Cancer Center, New York, NY 10065, USA. ${ }^{7}$ Ionis Pharmaceuticals, Carlsbad, CA 92010, USA. ${ }^{8}$ Present address: Department of Biochemistry, University of Otago, Dunedin 9016, New Zealand. ${ }^{9}$ These authors contributed equally: Sarah D. Diermeier, Allen T. Yu. ${ }^{\bowtie}$ email: spector@cshl.edu
} 
$\mathrm{B}$ reast cancer is the most common cancer among women in the United States and worldwide, with an estimated 276,480 new cases of invasive disease in women in the United States in $2020^{1}$. Although overall breast cancer mortality has been decreasing over the past two decades, it is still the second leading cause of cancer deaths in American women. According to the National Cancer Institute, there will be an estimated 42,170 deaths due to this disease in 2020, accounting for $15 \%$ of all cancer deaths among women. Breast tumors can be classified into multiple subtypes based on histological evaluation and the most frequent type of breast tumors are ductal carcinomas, which affect the milk ducts of the breast. Ductal carcinomas can further be separated into two groups: non-invasive ductal carcinoma in situ (DCIS), and invasive ductal carcinoma (IDC) which accounts for $75 \%$ of all breast cancers ${ }^{2,3}$. Breast cancer is recognized as a heterogeneous disease and molecular classification of invasive breast carcinomas can stratify tumors into informative subtypes and provide key prognostic signatures. In addition to traditional pathological characterization and immunohistochemistry (IHC) to examine protein levels of markers such as estrogen receptor (ER), progesterone receptor (PR), and epidermal growth factor receptor-2 (HER2), additional studies evaluating genomic rearrangements and molecular expression profiles of breast cancers have provided further genetic insights to better understand the disease ${ }^{4-6}$. These approaches have identified six major molecular subtypes of breast cancer (luminal A, luminal B, HER2-enriched, triple-negative/basal-like, normal breast-like, and claudin-low) ${ }^{7}$, each displaying different phenotypic and molecular features and which have distinct clinical outcomes.

In recent years, large scale genome-wide studies indicated that thousands of RNAs can be transcribed from the human and mouse genomes that lack protein-coding capacity ${ }^{8-10}$. In particular, long non-coding RNAs (lncRNAs) with a length $\geq 200$ nucleotides have been suggested to play key roles in a diverse range of biological processes ${ }^{11-13}$. Most lncRNAs are capped, spliced, and polyadenylated ${ }^{8}$. In addition, many lncRNAs are expressed in a tissue-specific and/or cell-type-specific manner and are involved in various gene-regulatory pathways ${ }^{14,15}$. Furthermore, misregulation of IncRNA expression has been linked to various diseases such as neuromuscular diseases, developmental disorders, neurodegenerative diseases, and cancers ${ }^{16-20}$. Several lncRNAs have been implicated as regulatory molecules in breast cancer progression and metastasis through different mechanisms $^{21,22}$. For example, the HOX antisense intergenic RNA (HOTAIR) is overexpressed in primary breast tumors and related to cancer progression and metastasis. HOTAIR can change the localization pattern of Polycomb repressive complex 2 (PRC2) and histone methylation to regulate gene expression in breast carcinoma cells ${ }^{23}$. Recent findings suggest that the lncRNA breast cancer anti-estrogen resistance $4(B C A R 4)^{24}$ can control GLI family zinc finger 2 (GLI2) gene expression to promote cancer cell migration by interacting with Smad nuclear interacting protein 1 (SNIP1) and serine/threonine-protein phosphatase 1 regulatory subunit 10 (PNUTS). Targeting BCAR4 by locked nucleic acids (LNA) in mouse models significantly affects cancer cell invasion and reduces lung metastases ${ }^{25}$. Genetic knockout or ASOmediated knockdown of Metastasis Associated Lung Adenocarcinoma Transcript 1 (Malat1) was shown to result in the differentiation of primary mammary tumors and a significant reduction in metastasis 26,27 . In addition to transcriptional regulation, lncRNAs can have other regulatory roles. For example, the lncRNA PVT1 has been shown to regulate Myc protein stability in breast cancer cells, resulting in cancer cell progression ${ }^{28}$, and the IncRNA NKILA can interact with and stabilize the NF$\kappa \mathrm{B} / \mathrm{I} \kappa \mathrm{B}$ complex and inhibit breast cancer metastasis ${ }^{29}$. However, for the majority of lncRNAs, the exact function and molecular mechanism of action in breast cancers still await detailed characterization. Previously, we performed RNA sequencing (RNA-seq) to identify differentially expressed lncRNAs between mammary tumor cells and normal mammary epithelial cells. From this screen, we identified 30 previously uncharacterized lncRNAs as Mammary Tumor Associated RNAs 1-30 (MaTARs 1-30) ${ }^{30}$.

Here, we examined the role of MaTAR25 in mammary tumor progression and metastasis. We found that genetic knockout (KO) of MaTAR25 in highly aggressive 4T1 triple-negative (ER-, $\mathrm{PR}-$, HER2-) mammary carcinoma cells results in a reduction in cell proliferation, migration, and invasion. KO cells transplanted into the mammary fat pad of $\mathrm{BALB} / \mathrm{c}$ mice results in a significant decrease in tumor growth as compared to $4 \mathrm{~T} 1$ control cells. Further, tail-vein injection of luciferase-labeled MaTAR25 $\mathrm{KO}$ cells showed reduced homing to the lungs and a significant decrease in metastatic nodules. In a complementary study, an antisense oligonucleotide (ASO)-mediated knockdown (KD) of MaTAR25 in the MMTV-Neu-NDL mouse model results in a significant decrease in tumor growth and a reduction in lung metastases. Analysis of the molecular function of MaTAR25 indicates that it regulates the Tns1 gene at the transcriptional level. Loss of MaTAR25 results in a reduction of Tns1 at the RNA and protein levels and a subsequent reorganization of the actin cytoskeleton and a reduction in focal adhesions and microvilli. Together, our data reveal MaTAR25, and its identified human ortholog LINC01271, as an exciting therapeutic candidate whose expression can be altered to impede breast cancer progression and metastasis.

\section{Results}

Characterization of MaTAR25, a nuclear-enriched lncRNA. We previously performed an RNA-seq screen to identify lncRNAs overexpressed in mammary tumors vs normal mammary epithelial cells as a means to identify potential candidates involved in mammary cancer progression and to explore their potential as therapeutic targets or key biomarkers in human breast cancer ${ }^{30}$. Among those lncRNA genes identified, the MaTAR25 gene on mouse chromosome 2 was originally annotated as 1200007C13Rik and it encodes a single transcript containing two exons (Fig. 1a). MaTAR25 is overexpressed in mammary tumors in the MMTVNeu-NDL (HER2 subtype) model compared to normal mammary epithelial cells and it is also upregulated in luminal and triplenegative subtypes of mammary cancer ${ }^{30}$. Analysis of ENCODE and FANTOM5 RNA-seq data has shown there is little to no expression of MaTAR25 in normal mouse tissues compared to MMTV-Neu-NDL tumor cells ${ }^{30,31}$ (Supplementary Fig. 1a). The full-length MaTAR25 transcript was determined to be 1978 nucleotides by $5^{\prime}$ and $3^{\prime}$ rapid amplification of cDNA ends (RACE) and Sanger sequencing (Fig. 1b), which was further confirmed by northern blot analysis (Fig. 1c).

According to three independent computational coding potential prediction programs, the MaTAR25 RNA transcript has very low protein-coding potential and is suggested to be a noncoding RNA (Supplementary Fig. 1b-d). However, there is one predicted open reading frame $(\mathrm{ORF})$ with the potential to generate a 123 amino acid peptide $(\sim 13 \mathrm{kDa})$. In order to assess whether a peptide is encoded by the MaTAR25 transcript, we performed in vitro transcription and translation. Compared to a luciferase DNA control (expected size $61 \mathrm{kDa}$ ) and a Xenopus laevis Histone H2B (HISTH2B) expressing plasmid control (expected size 14 $\mathrm{kDa}$ ), there was no detectable peptide generated from a plasmid that contained the MaTAR25 sequence (Fig. 1d). Together, these computational and experimental results confirm that MaTAR25 does not make a peptide, and thus is a bona fide lncRNA. 
a

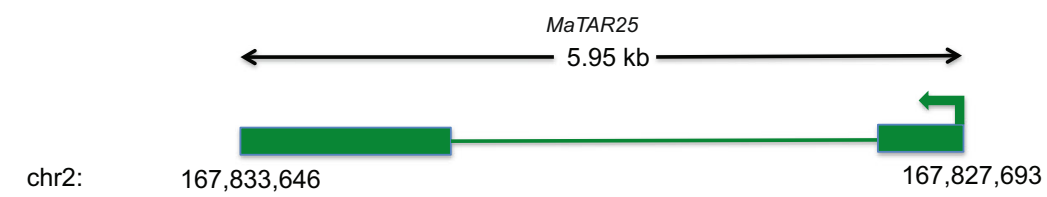

b
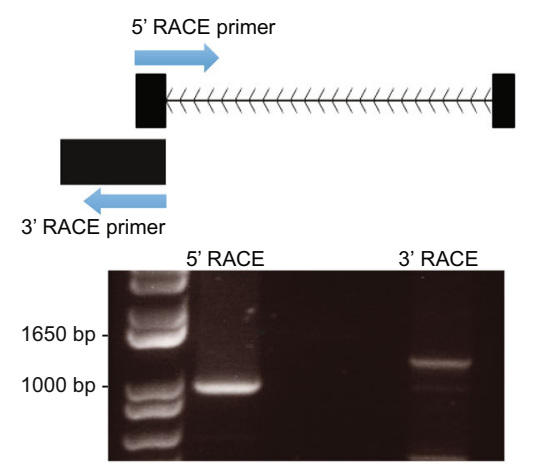

d

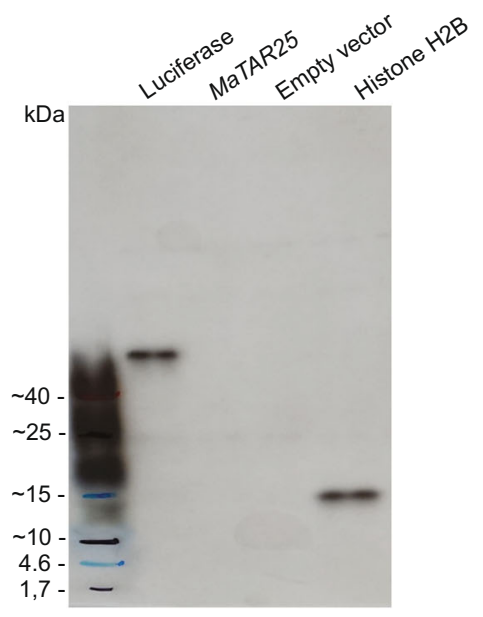

C

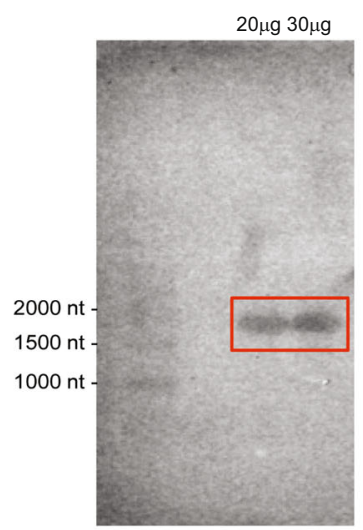

e

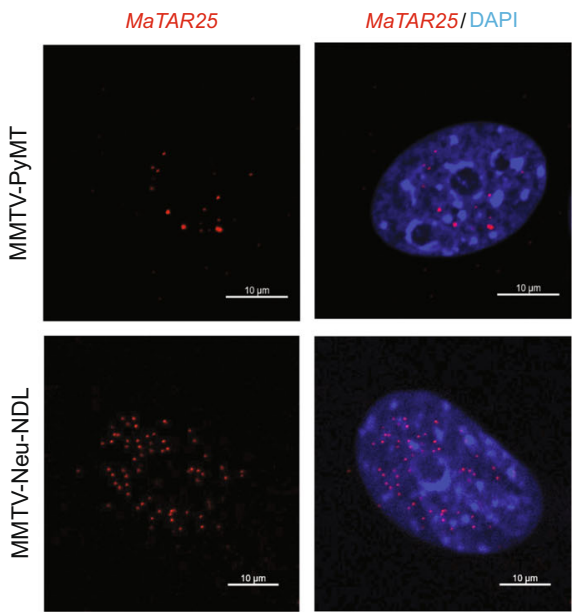

Fig. 1 Characterization of Mammary Tumor Associated RNA 25 (MaTAR25). a Representation of the MaTAR25 gene locus. MaTAR25 is an intergenic IncRNA gene located on mouse chromosome 2, and the MaTAR25 RNA transcript contains 2 exons and a poly (A) tail. b 5' and 3' rapid amplification of cDNA ends (RACE) was performed to identify the full-length MaTAR25 transcript. Three independent experiments were performed, and a representative gel image is shown. c The full-length MaTAR25 transcript was confirmed by northern blot analysis to be $\sim 2000 \mathrm{nt}$. In total, $20 \mu \mathrm{g}$ or $30 \mu \mathrm{g}$ of total RNA samples extracted from MMTV-PyMT primary cells were electrophoresed on a $1 \%$ agarose gel and probed. Two independent experiments were performed, and a representative gel image is shown. $\mathbf{d}$ In vitro transcription and translation reactions were performed to confirm that MaTAR25 does not produce a peptide. The reaction products were loaded on a 4-20\% gradient SDS-PAGE gel, and the signals were detected by HRP-conjugated streptavidin. Luciferase control DNA and Xenopus laevis Histone H2B (HISTH2B) expressing plasmids were used as positive controls and empty vector as a negative control. Three independent experiments were performed, and a representative gel image is shown. e Representative smRNA-FISH images showing localization of MaTAR25 RNA transcripts (red) within nuclei of MMTV-PyMT and MMTV-Neu-NDL primary cells. Scale bars are $10 \mu \mathrm{m}$.

In order to determine the localization and abundance of MaTAR25, we performed single-molecule RNA fluorescence in situ hybridization (smRNA-FISH) to detect MaTAR25 RNA transcripts within MMTV-PyMT (luminal B) and MMTV-NeuNDL (HER2/neu+) primary mammary tumor cells. The majority of MaTAR25 transcripts were detected in cell nuclei (Fig. 1e), and each nucleus contained $\sim 10-15$ transcript foci. Thus, MaTAR25 is a nuclear-enriched lncRNA with a potential role in the regulation of gene expression in mammary cancer cells.
MaTAR25 knockout decreases 4T1 cell viability/migration/ invasion. To assess the functional role of MaTAR25, we proceeded to genetically KO MaTAR25 in highly aggressive 4T1 triple-negative (ER-, PR-, HER2-) mammary carcinoma cells using CRISPR/ Cas9. We designed gRNA pairs targeting various regions upstream and downstream of the transcription start site (TSS) of MaTAR25 to create a genomic deletion (Fig. 2a and Supplementary Fig. 2a). MaTAR25 knockout clones were single-cell sorted and selected by Sanger sequencing, qRT-PCR, as well as single-molecule RNA-FISH (Fig. 2a and Supplementary Fig. 2b). 
a

\begin{tabular}{|l|l|l|l|l|l|l|l|l|l|l|l|}
\hline 2qE3 & E4 & $2 \mathrm{qE} 5$ & $2 \mathrm{qF} 1$ & $\mathrm{~F} 2$ & $2 \mathrm{qF} 3$ & $2 \mathrm{qG} 1$ & $\mathrm{G} 3$ & $2 \mathrm{qH} 1$ & $\mathrm{H} 2$ & $2 \mathrm{qH} 3$ & $2 \mathrm{qH} 4$ \\
\hline
\end{tabular}

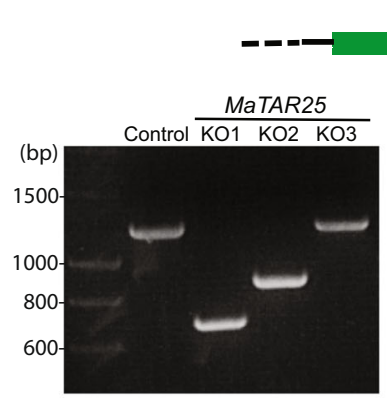

b

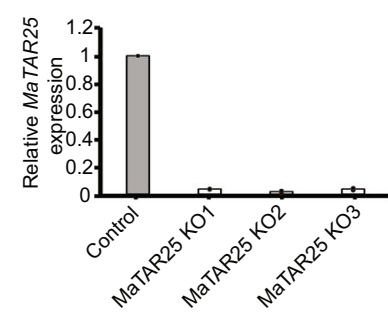

C

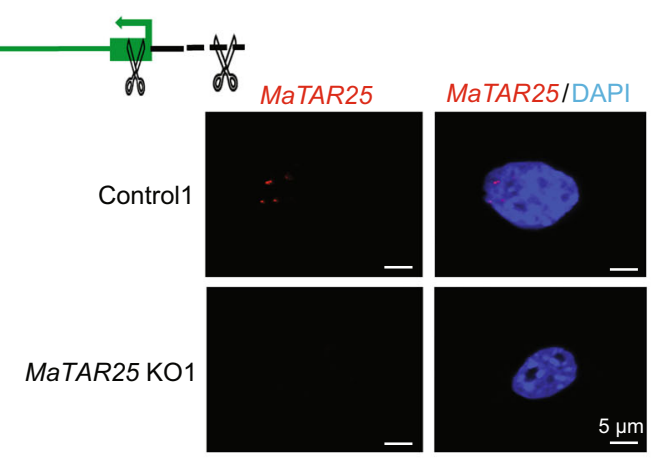

d

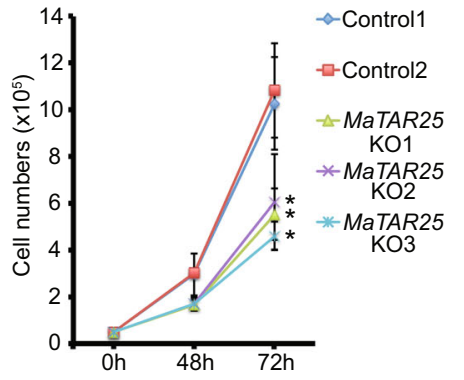

e

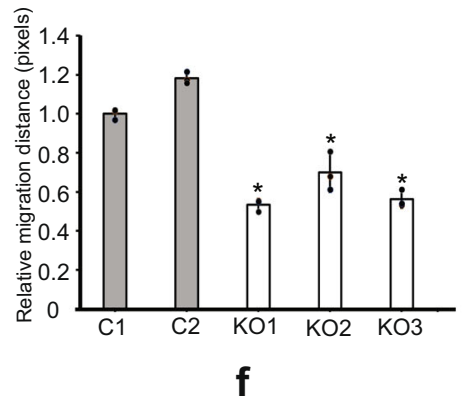

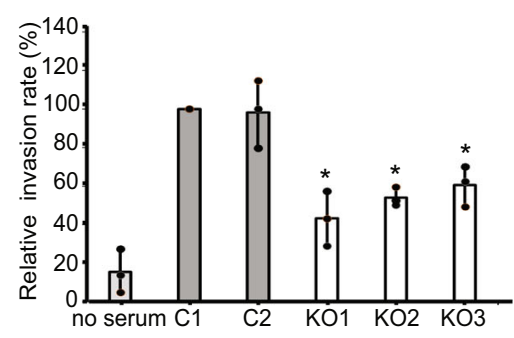
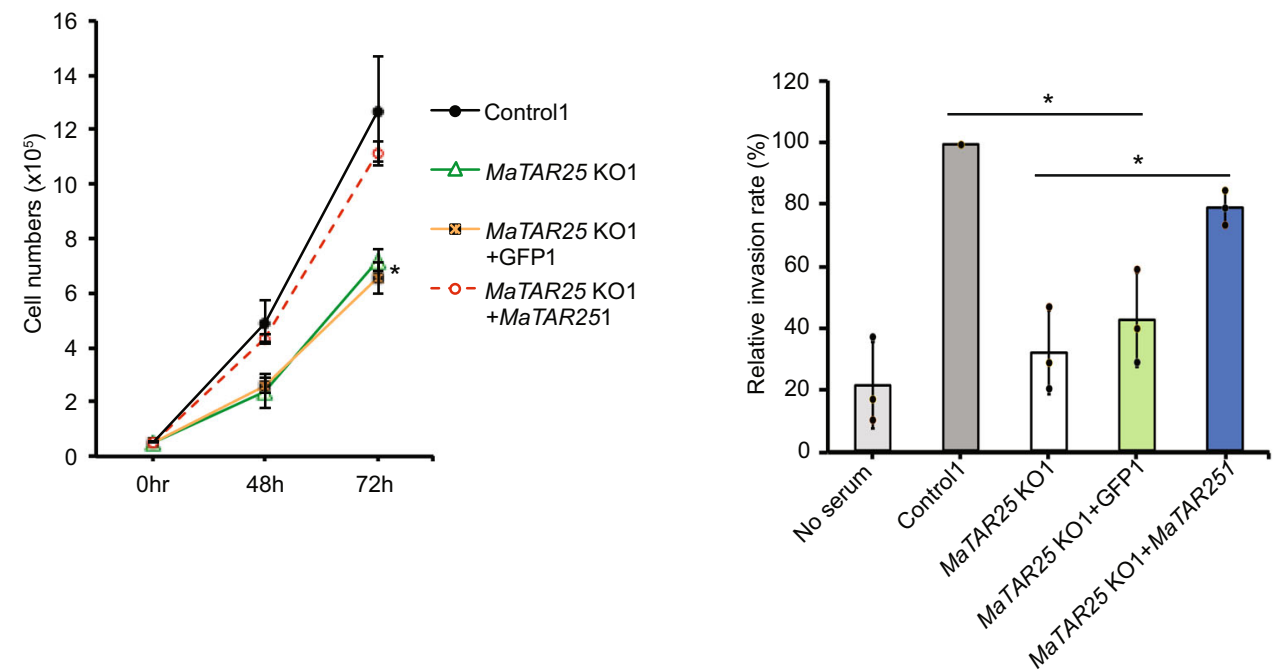

Fig. 2 MaTAR25 knockout affects 4T1 cell viability, migration, and invasion in vitro; all of which can be rescued by ectopic expression of MaTAR25 in knockout cells. a CRISPR/Cas9 was used to generate MaTAR25 KO clones in 4T1 cells. Pairs of sgRNAs were introduced targeting upstream and downstream of the transcription start site of MaTAR25, resulting in 390-620 bp genomic deletions over this region, and a Renilla Luciferase sgRNA was used as a negative control. Knockout clones were selected by genomic PCR and Sanger sequencing for homozygous genomic deletion. Control clones were selected by qRT-PCR for expressing similar levels of MaTAR25 as parental 4T1 cells. QRT-PCR $(n=2)$, and representative images of single smRNA-FISH (red: MaTAR25) are shown to confirm MaTAR25 KO. Scale bars are $5 \mu \mathrm{m}$. b $4 \mathrm{~T} 1$ cells were seeded at the same cell density in 12-well tissue culture plates at day 0 , and cell counting was performed at different time points. The mean cell numbers of three independent replicates of $4 \mathrm{~T} 1$ control groups and MaTAR25 KO groups are shown \pm SD $(n=$ 3). ${ }^{\star} P<0.05$ (paired Student's $t$ test; two-tailed). c Live-cell tracking was performed over time to examine cell migration. Images were collected every 5 min for a total of $8 \mathrm{~h}$ and analyzed by CellTracker image-processing software. The mean relative migration distance ( $\mu \mathrm{m})$ of $4 \mathrm{T1}$ control groups and MaTAR25 KO groups is shown $\pm \mathrm{SD}$ ( $n=3$ independent replicates). ${ }^{*} P<0.05$ (paired Student's $t$ test; two-tailed). d Twenty-four-well Boyden chamber invasion assay ( $24 \mathrm{~h}$ ). The mean relative cell invasion of three independent replicates of 4 T1 control groups and MaTAR25 KO groups is shown \pm SD $(n=3)$. * $P<0.05$ (paired Student's $t$ test; two-tailed). e Selected clones with ectopic expression of MaTAR25 or GFP were used as positive and negative controls to assess rescue in a cell viability assay. Data are presented as mean values \pm SD $(n=3)$. ${ }^{\star} P<0.05$ (paired Student's $t$ test; two-tailed). $\mathbf{f}$ Cell invasion assay. The mean cell numbers and mean relative cell invasion of three independent replicates of 4T1 control1, MaTAR25 KO1, MaTAR25 KO1 with GFP expression, and MaTAR25 KO1 with MaTAR25 ectopic expression is shown \pm SD $(n=3)$. ${ }^{\star} P<0.05$ (paired Student's $t$ test; two-tailed). 
After selecting several MaTAR25 KO clones, we evaluated them for alterations in cell viability, migration, and invasion as compared to control 4T1 cells. MaTAR25 KO cells exhibited a significant decrease of $50 \%$ in cell viability as compared to $4 \mathrm{~T} 1$ control cells (Fig. 2b). To further investigate this phenotype, we performed BrdU labeling and FACS analysis, which demonstrated a twofold increase in G2 cells, suggesting that the decreased proliferation phenotype is most likely the result of a lengthened G2 phase (Supplementary Fig. 2c). As cell migration and invasion are critical processes associated with metastasis, we were interested in determining whether MaTAR25 loss might play a role in these events. We used a live-cell tracking assay to assess cell migration and we found a $40 \%$ reduction in cell motility upon loss of MaTAR25 (Fig. 2c, Supplementary Fig. 2d, and Supplementary Movies 1, 2). A wound-healing assay also corroborated the observed difference in cell migration between 4T1 control and MaTAR25 KO cells (Supplementary Fig. 2e). Finally, we used a Boyden chamber invasion assay and found that loss of MaTAR25 resulted in a $45 \%$ reduction in invasion ability as compared to $4 \mathrm{~T} 1$ control cells (Fig. 2d).

In order to exclude the possibility that the phenotypes observed in MaTAR25 KO cells were caused by disturbing chromatin structure rather than specific loss of the MaTAR25 transcript, we generated single-cell ectopic overexpression clones of MaTAR25 in 4T1 MaTAR25 KO cells. Ectopic expression of MaTAR25 rescued both the cell proliferation and invasion phenotypes (Fig. 2e, f), indicating that MaTAR25 RNA plays an important role in these processes in situ, and likely exhibits its effect in trans. Hence, MaTAR25 appears to be an important lncRNA impacting mammary tumor cell growth and critical aspects of metastasis. To further explore MaTAR25's downstream targets, we performed RNA-seq to identify differentially expressed genes by comparing MaTAR25 KO cells with $4 \mathrm{~T} 1$ control cells (Supplementary Data 1). Pathway analysis of the differentially expressed genes by Kyoto Encyclopedia of Genes and Genomes (KEGG) and Gene Set Enrichment Analysis (GSEA) both revealed alteration in cell cycle and DNA-related processes, both related to the observed phenotypes we observed in MaTAR25 KO cells (Supplementary Fig. 2f). The expression levels of three neighboring proteincoding genes (Snail, Cebpb, and Ptpn1) were not consistently altered in MaTAR25 KO cells compared to 4T1 control cells (Supplementary Fig. 2g). In addition, the expression levels of these three neighboring protein-coding genes were not significantly changed upon MaTAR25-specific antisense oligonucleotide (ASO)-mediated knockdown in $4 \mathrm{~T} 1$ and $\mathrm{cNeu}$ (MMTV-Neu-NDL) cells consistent with the role of MaTAR25 functioning in trans (Supplementary Fig. 2h).

MaTAR25 knockout decreases tumor progression/metastasis in vivo. In order to further evaluate the functional impact and the therapeutic potential of MaTAR25 in mammary tumor progression, we performed multiple in vivo studies. Injection of MaTAR25 4T1 KO cells into the mammary fat pad of BALB/c mice resulted in a significant $56 \%$ decrease in tumor growth at day 28, compared to the $4 \mathrm{~T} 1$ control injected group (Fig. 3a, b). In addition, we performed tail-vein injection using MaTAR25 KO cells expressing a luciferase reporter to track cancer cell homing and metastasis to the lungs in $\mathrm{BALB} / \mathrm{c}$ mice. The in vivo bioluminescence signal in the lungs of mice injected with MaTAR25 $\mathrm{KO}$ cells was reduced (Supplementary Fig. 3a) compared to those injected with $4 \mathrm{~T} 1$ control cells. On day 21, the number of metastatic nodules in lung samples collected from the MaTAR25 $\mathrm{KO}$ group was also significantly decreased by $62 \%$ compared to the 4T1 control group (Fig. 3c).
As a complementary approach to CRISPR/Cas9 KO, we designed a series of MaTAR25-specific antisense oligonucleotides (ASOs), (16-mers) comprised of phosphorothioate-modified short S-cEt (S-2'-O-Et-2',4'-bridged nucleic acid) gapmer chemistry ${ }^{32-34}$. We individually screened multiple ASOs targeting MaTAR25 to identify the most effective ASOs in terms of KD efficiency by qRT-PCR after $48 \mathrm{~h}$ and $72 \mathrm{~h}$ of ASO treatment in $4 \mathrm{~T} 1$ cells. The two most effective MaTAR25 ASOs achieved a knockdown ranging from 70 to $90 \%$ (Supplementary Fig. 1e). When comparing MaTAR25 ASO-treated cells to mock or scrambled ASO (scASO)-treated $4 \mathrm{~T} 1$ control cells after $72 \mathrm{~h}$, we found a significant decrease in cell viability using cell counting assays ( $-45 \%$ for ASO1 and $-38 \%$ for ASO2) (Supplementary Fig. 1f), and similar reduction phenotypes were also observed in cNeu (MMTV-Neu-NDL) cells treated with MaTAR25 ASOs ( $-50 \%$ for ASO1 and $-41 \%$ for ASO2) (Supplementary Fig. 1g), consistent with our $\mathrm{KO}$ studies indicating that MaTAR25 has a role in mammary cancer cell proliferation.

Furthermore, to assess the therapeutic potential of reducing the level of MaTAR25 in vivo, we evaluated the impact of subcutaneous injection of two independent MaTAR25 ASOs for their in vivo ability to knockdown MaTAR25 and to impact mammary tumor progression in the MMTV-Neu-NDL mouse model. ASO-mediated knockdown of MaTAR25 resulted in a $59 \%$ decrease in tumor growth compared to the scASO control group (Fig. 3d and Supplementary Fig. 3b). By comparing the hematoxylin and eosin (H\&E)-stained tumor sections collected from the MaTAR25 ASO-injected group with the scASO control group, we observed a strong level of necrosis in the MaTAR25 ASO-treated mammary tumor samples (Fig. 3e) but not in other non-tumor tissues (Supplementary Fig. 3c). Importantly, mammary tumors from the scASO control group lacked any significant necrotic phenotype (Fig. 3e). We also collected lung samples from each group to examine for the presence of micrometastases, and the H\&E-stained lung sections showed that KD of MaTAR25 resulted in a $40 \%$ incidence rate of micro-metastatic nodules in lungs from ASO1 or ASO2-treated animals as compared to a $76.9 \%$ incidence rate for the scASO control group (Supplementary Fig. 3d). Together, our in vitro and in vivo data indicate that MaTAR25 plays a critical role in promoting mammary tumor progression and metastasis.

MaTAR25 is a positive upstream regulator of Tns1, a mediator of cell-matrix adhesion and migration. Next, we were interested in revealing aspects of the molecular mechanism of action of MaTAR25 in regulating mammary tumor progression. Since we previously identified MaTAR25 to be highly enriched in cell nuclei by smRNA-FISH, we went on to perform cell fractionation to isolate cytoplasmic and nucleoplasmic lysates as well as chromatin pellets of $4 \mathrm{~T} 1$ cells to determine the subcellular enrichment of MaTAR25 by qRT-PCR analysis. Notably, compared to the enrichment of $\beta$-actin and Malat1, we found a significant enrichment of MaTAR25 in the nucleoplasmic and chromatin fractions (Fig. 4a), indicating that the molecular mechanism of action of MaTAR25 may be related to transcriptional regulation. To test this hypothesis, we performed Chromatin Isolation by RNA Purification (ChIRP) ${ }^{35}$ to pull down RNA/DNA complexes by using specific biotin-labeled antisense oligonucleotides targeting MaTAR25 as well as biotin-labeled antisense oligonucleotides targeting housekeeping gene Ppib transcripts as the corresponding control (Fig. 4b). ChIRP-seq identified MaTAR25 genomic targeting sites (Supplementary Data 2,3), and revealed that these targets are highly enriched in simple repeats regions and LTRs (log ratio enrichments to input are two- to threefold). According to Hypergeometric 
a

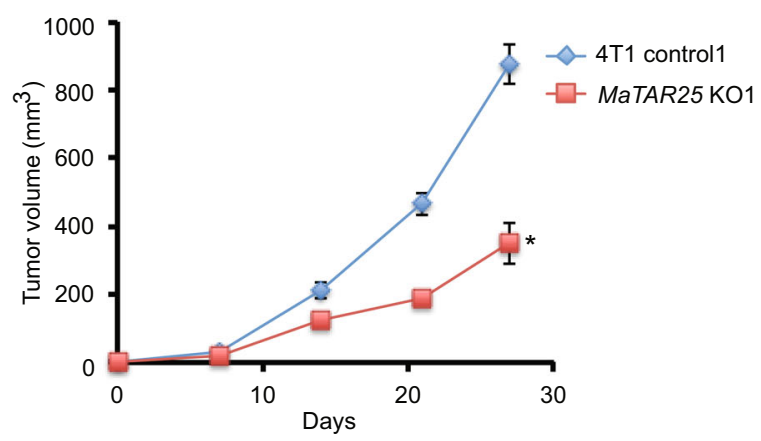

C

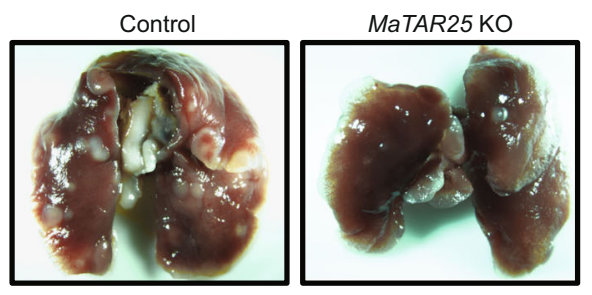

b
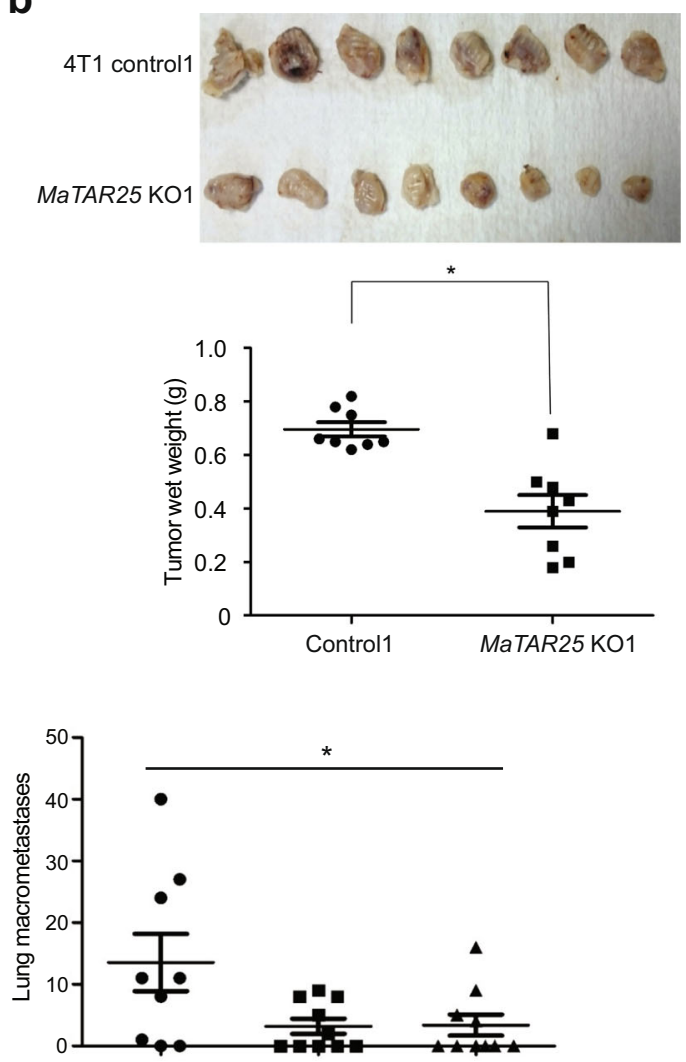

e

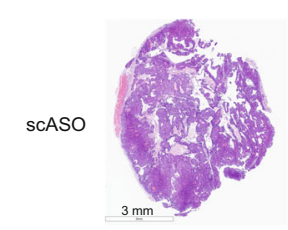

$\rightarrow$ SCASO

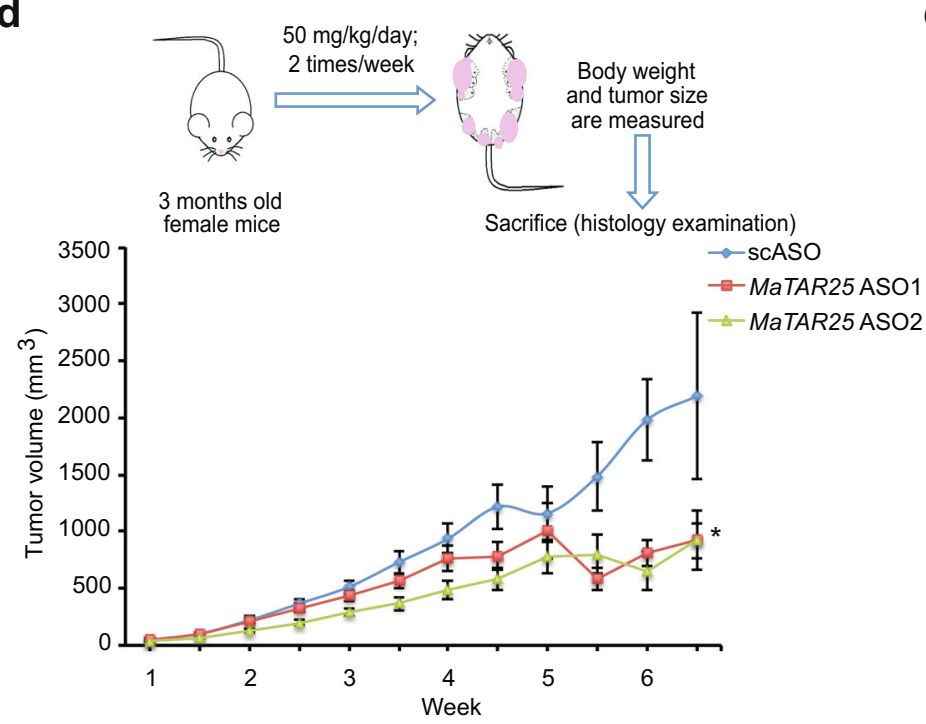

and tumor size

are measured

[]

Fig. 3 MaTAR25 knockout impairs tumor growth and metastasis in vivo. a 4T1 control or MaTAR25 KO cells were injected orthotopically into the mammary fat pad of female BALB/c mice. Primary tumors were measured every week over a period of 4 weeks, and the mean tumor volume of eight mice per group is shown \pm SE. ${ }^{\star} P<0.05$ (paired Student's $t$ test; two-tailed). $\mathbf{b}$ Ten mice were sacrificed, and tumors were collected at day 28 to compare the tumor growth rate between the control group and MaTAR25 KO groups. Tumors derived from MaTAR25 KO cells showed a 56\% reduction in tumor growth. The mean tumor wet weight is shown \pm SE. ${ }^{*} P<0.05$ (paired Student's $t$ test; two-tailed). c Female BALB/c mice were injected into the tail vein with 4T1 control1 or MaTAR25 KO cells. Mice were monitored every week and sacrificed at day 21. Mouse lungs were collected and imaged (left panel), and lung metastatic nodules were counted to compare the metastatic ability between the control group and MaTAR25 KO group (right panel). Mice injected with MaTAR25 KO cells exhibited a 62\% reduction in lung metastatic nodules. Data are presented as mean values shown \pm SD. ${ }^{\star} P<0.05$ (paired Student's $t$ test; two-tailed). d Schematic showing the approach for ASO-mediated knockdown of MaTAR25 in MMTV-Neu-NDL mice. Two independent MaTAR25 ASOs or a control scASO were used for subcutaneous injection. Primary tumors were measured twice per week, and the mean tumor volume of seven mice per group is shown \pm SE. ${ }^{\star} P<0.05$ (paired Student's $t$ test; two-tailed). e Hematoxylin and eosin (H\&E)-stained tumor images showing the different histological phenotypes between tumor samples from the scASO-injected group and MaTAR25 ASO-injected group. More than five tumor samples in each group were examined, and representative images are shown. Scale bars are $3 \mathrm{~mm}$. 
a

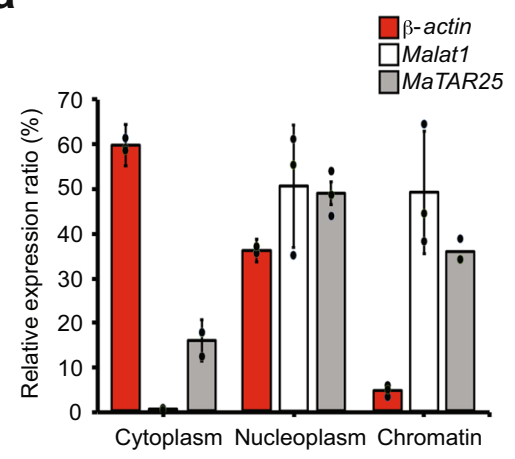

C

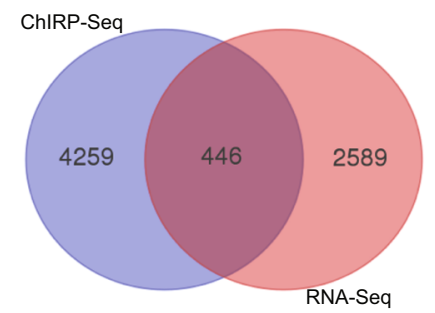

MaTAR25 odd \& even oligo pools overlapping targets

\begin{tabular}{|c|c|c|c|}
\hline \multicolumn{4}{|c|}{ Overlapping targets } \\
\hline 2 & MaTAR25 & 0.219515585 & $8.34 \mathrm{E}-04$ \\
\hline 1 & Tns1 & 0.379721873 & 0.06155137 \\
\hline 15 & Pvt1 & 0.624304762 & 0.02537443 \\
\hline 8 & Lonp2 & 0.625035638 & 0.01057177 \\
\hline 15 & Zhx2 & 0.428373444 & $1.38 \mathrm{E}-05$ \\
\hline 11 & Ggnbp2 & 0.653081803 & 0.08361245 \\
\hline 10 & Ilvbl & 0.755908045 & 0.08189884 \\
\hline 12 & Immp21 & 0.57074968 & 0.02643153 \\
\hline 7 & Cebpg & 0.360909244 & $1.85 \mathrm{E}-03$ \\
\hline
\end{tabular}

f

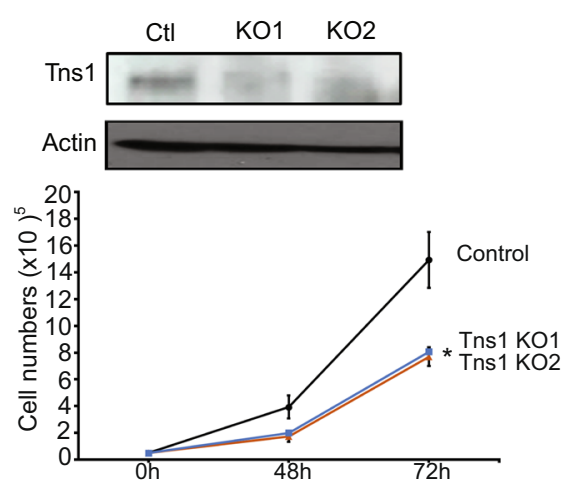

b

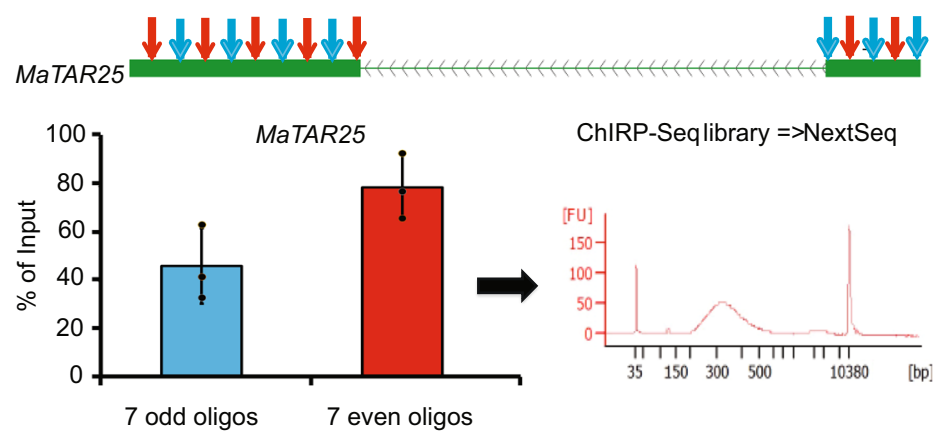

d

e
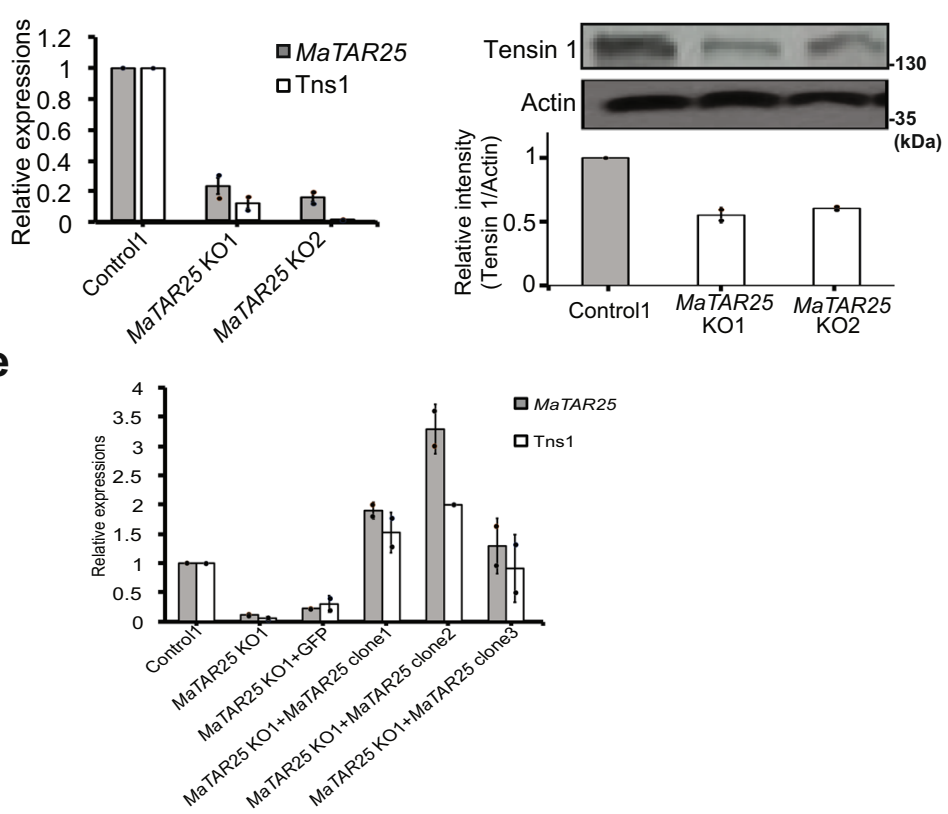

g

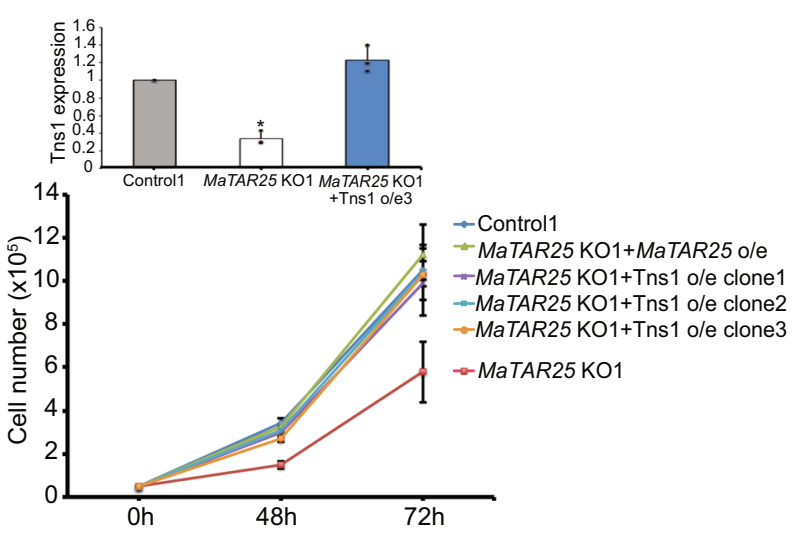

Optimization of Motif EnRichment (HOMER) motif analysis, these targets are potential binding sites of the transcription factors ZBTB7C, ZNF354C, Irf3, GATA1, and REL (Supplementary Fig. 4a). Combining the MaTAR25 KO RNA-seq data and ChIRP-seq results, we found a total of 446 overlapping genes (Fig. $4 \mathrm{c}$ and Supplementary Data 4), which could be downstream targets regulated by MaTAR25. Among these overlapping genes, the top gene ranked by ChIRP-seq data, just under MaTAR25 itself, is Tensin1 (Tns1) (Fig. 4c and Supplementary Fig. 4b). The Tns1 gene encodes for a protein that localizes to focal adhesions and positively regulates cell migration and invasion ${ }^{36,37}$. By qRTPCR and immunoblot analysis, we found that the RNA and protein levels of Tns1 are significantly lower in MaTAR25 KO cells than in $4 \mathrm{~T} 1$ control cells (Fig. 4d). Additional qRT-PCR results from MaTAR25 ASO1-mediated KD in 4T1 and cNeu cells also indicated Tns1 expression levels correlate with MaTAR25 RNA levels (Supplementary Fig. 4d). Interestingly, ectopic expression of MaTAR25 in MaTAR25 KO cells results in a corresponding increase in the level of Tns1 (Fig. 4e). Hence, we conclude that Tns1 is a direct downstream target of MaTAR25 
Fig. 4 MaTAR25 is a positive upstream regulator of Tns1. a Cell fractionation was performed to isolate cytoplasmic, nucleoplasmic, and chromatinassociated RNA. qRT-PCR was used to determine the subcellular localization ratio of MaTAR25 transcripts. $\beta$-actin and Malat 1 were used as marker RNAs for quality control. Data are presented as mean values \pm SD $(n=3$ independent experiments). $\mathbf{b}$ Schematic diagram showing the targeting of biotin-labeled oligonucleotides binding MaTAR25 transcripts for Chromatin Isolation by RNA Purification (ChIRP)-seq. Odd and even oligo pools (seven oligos in each pool) were used, and qRT-PCR was performed to check the RNA purification efficiency. Data are presented as mean values \pm SD ( $n=3$ independent experiments). c Venn diagram showing differentially expressed genes in MaTAR25 KO cells identified from RNA-Seq overlapped with MaTAR25 ChIRP-seq data. The top candidate genes are listed. Statistics were determined using DESeq2, and a FDR-adjusted $P$ value of $<0.1$ was set as a threshold for statistical significance. $\mathbf{d}$ Validation of Tns1 as a MaTAR25-targeted gene by qRT-PCR and immunoblotting in 4T1 control and MaTAR25 KO cells ( $n=2$ independent experiments). e The RNA expression level of Tns1 is rescued upon ectopic expression of MaTAR25 in MaTAR25 KO cells as determined by qRT-PCR ( $n=2$ independent experiments). f CRISPR/Cas9 targeting was used in 4T1 cells to generate Tns 1 knockout clones. The upper panel shows expression levels of Tns1 in $4 \mathrm{~T} 1$ control, Tns1 KO clone1, and Tns $1 \mathrm{KO}$ clone2 by immunoblotting. The lower panel shows the cell counting viability assay result of $4 \mathrm{~T} 1$ control1, Tns1 KO1, and Tns1 KO2. Results are mean \pm SD ( $n=3$ independent experiments). ${ }^{\star} P<0.05$ (paired Student's $t$ test; two-tailed). $\mathbf{g}$ Ectopic expression of Tns 1 in MaTAR25 KO cells rescues the cell viability defect. The top panel shows expression levels of Tns1 in 4T1 control1, MaTAR25 KO1, MaTAR25 KO1 with Tns1 ectopic expression clone3 by qRT-PCR. Results are mean \pm SD ( $n=3$ independent experiments). ${ }^{\star} p<0.05$ (paired Student's $t$ test; two-tailed). The bottom panel shows the cell counting viability assay results of 4T1 control1, MaTAR25 KO1, MaTAR25 KO1 with MaTAR25 ectopic expression, and MaTAR25 KO1 with Tns1 ectopic expression clone1-3. Results are mean \pm SD ( $n=2$ independent experiments).

and further confirming that MaTAR25 imparts its function in trans. In order to confirm the ChIRP-seq result and to further investigate how MaTAR25 might regulate the level of Tns1, we next performed double-label DNA FISH to detect the MaTAR25 (Chr2) and Tns1 (Chr1) gene loci in cells, and we found no physical interaction between these genomic loci (Supplementary Fig. 4e, upper panels). However, combined MaTAR25 smRNAFISH and Tns1 DNA FISH in the same cells showed that MaTAR25 RNA was overlapping with at least one Tns1 allele in $50 \%$ of the cells (Supplementary Fig. 4e). In contrast, RNA-FISH to localize MaTAR26 only showed a $14 \%$ overlap between the MaTAR26 RNA signal and a Tns1 allele (Supplementary Fig. 4e). This demonstrates the specificity of MaTAR25 RNA transcripts associating with the Tns1 gene to regulate its expression. We, therefore, performed CRISPR/Cas9 knockout using gRNAs targeting Tns 1 in $4 \mathrm{~T} 1$ cells and selected Tns $1 \mathrm{KO}$ clones for in vitro functional assays. We found that the Tns $1 \mathrm{KO}$ cells phenocopied the MaTAR25 KO cells and exhibited a significant $40 \%$ decrease in cell viability (Fig. 4f) and a 30\% decrease in cell migration vs control cells (Supplementary Fig. 4f). In addition, ectopic expression of Tns1 in 4T1 MaTAR25 KO cells can rescue the cell viability phenotype (Fig. $4 \mathrm{~g}$ ). Interestingly, high expression of TNS1 is strongly correlated with poor survival of grade 3 breast cancer patients $^{38}$ (Supplementary Fig. 4c). Together, these data indicate that Tns1 is a critical downstream target of MaTAR25.

Since Tns 1 is a key component of focal adhesion complexes and is responsible for cell-cell and cell-matrix interactions as well as cell migration by interacting with actin filaments ${ }^{39}$, we examined the organization of actin filaments, as well as the additional focal adhesion complex components paxillin and vinculin $^{40}$, in $4 \mathrm{~T} 1$ control and MaTAR25 KO cells by immunofluorescence (IF) confocal microscopy. Indeed, the F-actin microfilaments are disrupted (Supplementary Fig. 4g), and the distribution of paxillin and vinculin proteins are altered dramatically (Supplementary Fig. 4i) in 4T1 MaTAR25 KO cells as compared to $4 \mathrm{~T} 1$ control cells. Interestingly, both ectopic expression of MaTAR25 or Tns1 in 4T1 MaTAR25 KO cells can rescue the actin filament phenotype (Supplementary Fig. $4 \mathrm{~g}$ ), supporting our finding that Tns1 is a critical downstream target of MaTAR25 regulating mammary tumor progression. To further evaluate the phenotype of MaTAR25 KO cells, we used transmission electron microscopy (TEM) (Supplementary Fig. 4h). TEM clearly revealed a dramatic reduction of microvilli over the cell surface of MaTAR25 KO cells compared to 4T1 control cells, indicating loss of MaTAR25 expression impacts the actin-bundling process (Supplementary Fig. 4h) as well as microvilli formation in $4 \mathrm{~T} 1$ cells.
MaTAR25 interacts with PURB to carry out its function. It has been suggested that lncRNAs can interact with transcriptional regulators/co-factors to form ribonucleoprotein (RNP) complexes to regulate the expression of downstream genes in the cell nucleus $^{41}$. To identify MaTAR25 interacting proteins, we used two different paired sets of biotin-labeled antisense oligonucleotides targeting MaTAR25 for native RNA antisense oligonucleotide pulldown (RAP) in 4T1 cells followed by qRT-PCR which revealed a 50-60\% pull-down efficiency (Supplementary Fig. 5a). Samples were eluted from beads for mass spectrometry isobaric tags for relative and absolute quantitative (MS-iTRAQ) analysis to identify proteins that bind to MaTAR25, and Ppib as the corresponding control. We ranked the candidate interactors based on detectable peptides above background in both pair sets of oligonucleotide pull-downs, and selected candidates with at least twofold enrichment compared to corresponding Ppib oligo pulldown (Fig. 5a and Supplementary Data 9). Among the protein candidates, two transcription co-regulators always appeared on the top list between multiple runs. These are purine-rich element-binding protein A (PURA) and purine-rich elementbinding protein $\mathrm{B}$ (PURB), which can form homodimers or heterodimers in the nucleus ${ }^{42}$. Additionally, one other protein, $\mathrm{Y}$ box protein 1 (YBX1) also on the candidate list, but which did not pass the enrichment criteria, was shown in a previous study to interact with PURA to form a PURB/PURA/YBX1 heterotrimer $^{43}$. To verify our MS result, we first performed immunoblot analysis with PURA and PURB antibodies and we could detect significantly higher enrichment of PURA and PURB in samples of MaTAR25 oligonucleotide pulldown than in the Ppib oligonucleotide pulldown (Fig. 5b, upper panel). RNA immunoprecipitation (RIP) using PURB antibodies compared to IgG control also revealed the specificity of the MaTAR25-PURB interaction (Fig. 5c) but not MaTAR25-PURA (Supplementary Fig. 5b). Based on these data, we demonstrate that PURB is the lead protein directly binding to MaTAR25. Immunoblot analysis using pulldown samples from 4T1 and 4T1 MaTAR25 KO cell lysates (Fig. 5b, lower panel) plus pull-down samples from NDL primary cell lysates (Supplementary Fig. 5c) confirmed the specific interaction between MaTAR25 and PURB. To further confirm the role of PURB in regulating Tns1, we manipulated the level of PURB in 4T1 cells either through ectopic overexpression (Fig. 5d) or siRNA-mediated knockdown and demonstrated upregulation or downregulation of Tns1, respectively (Supplementary Fig. 5d). The results confirmed that the expression level of Tns1 is related to the changes in PURB expression level in $4 \mathrm{~T} 1$ cells, indicating that the MaTAR25/PURB RNP complex is essential for the regulation of Tns1. 
a

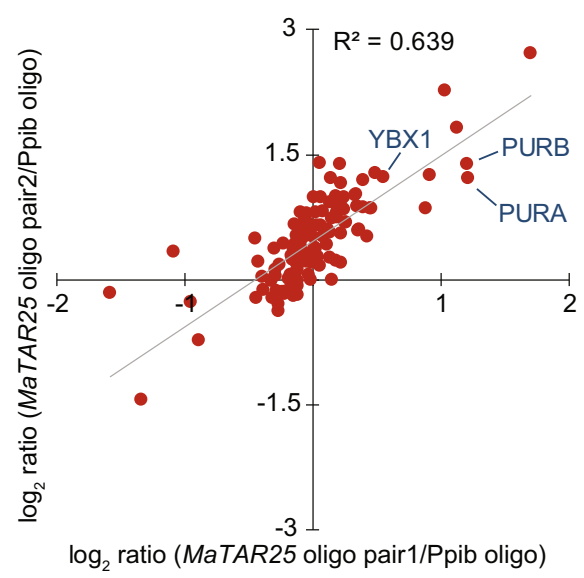

C

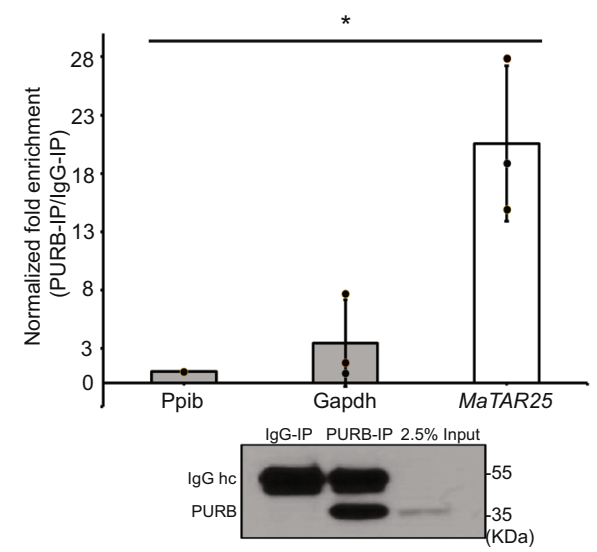

e

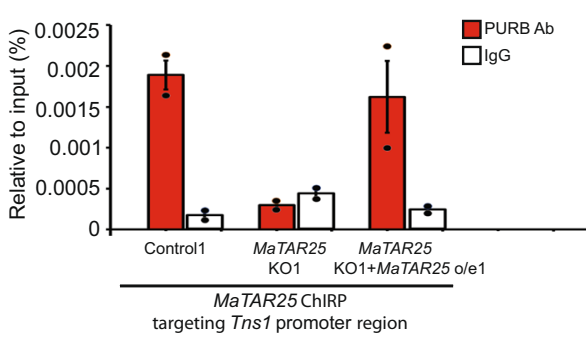

b

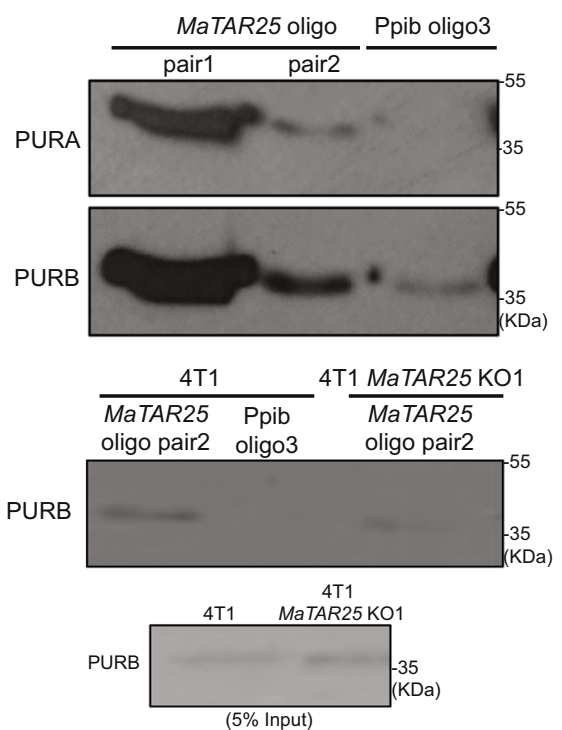

d

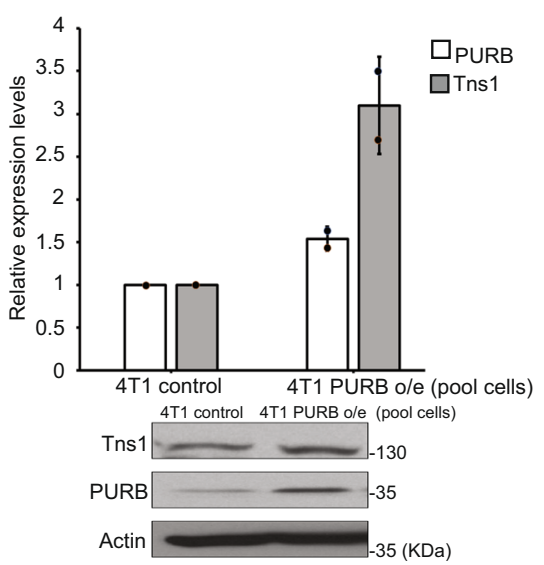

Fig. 5 MaTAR25 interacts with PURB to carry out its function. a Scatterplot depicts the fold enrichment of protein candidates from isobaric tags for the relative and absolute quantitation (iTRAQ) analysis comparing two independent oligo pair sets targeting MaTAR25 RNA transcripts vs Ppib RNA transcripts. b Upper: immunoblot analysis of PURA and PURB following pulldown of MaTAR25 or Ppib from 4T1 cells. Lower: immunoblot analysis of PURB following the pulldown of MaTAR25 or Ppib from 4T1 cells or 4T1 MaTAR25 KO cells. More than three different experiments were performed, and representative images are shown. c MaTAR25, Ppib, and Gapdh transcripts were assessed by qRT-PCR in endogenous PURB, or IgG (negative control) immunoprecipitates from $4 \mathrm{~T} 1$ cells. Fold enrichment of PURB associated RNA signal over IgG signal is calculated and data are presented as mean values \pm SD $\left(n=3\right.$ independent experiments). ${ }^{*}<0.05$ (paired Student's $t$ test; two-tailed). Immunoblot analysis of PURB was performed as a control. $\mathbf{d}$ qRT-PCR analysis and immunoblotting of Tns1 expression in 4T1 cells following ectopic overexpression of PURB. The relative expression levels are shown as mean values \pm SD ( $n=2$ independent experiments). e ChIP-qPCR analysis of PURB occupancy over the identified MaTAR25 targeting region and non-targeting region of the Tns1 DNA locus by ChIRP-seq analysis. ChIP-qPCR was performed in 4T1 control1 cells, 4T1 MaTAR25 KO1 cells, and upon ectopic expression of MaTAR25 in MaTAR25 KO1 cells. Primers for a MaTAR25 non-targeting region and the Gapdh TSS were used as negative controls. Bar graphs represent the mean $\pm S D$ ( $n=2$ independent experiments). 
Tns1 isoform 3 was identified as the major isoform expressed in MMTV-Neu-NDL and 4T1 cells (Supplementary Fig. 5e). Based on our ChIRP-seq result, we were able to go on to identify the promoter region of Tns 1 isoform 3, which contains a very high purine:pyrimidine ratio (3:1) including many potential PURB-binding sequence motifs (GGTGG) 44 , as the main targeting region in the Tns1 gene (Supplementary Fig. 5f). Moreover, Hypergeometric Optimization of Motif EnRichment (HOMER) motif analysis based on ChIRP-seq data indicated the top enriched motif sequence of MaTAR25 interacting genes is GGTGGTGGAGAT further supporting the MaTAR25-PURBbinding motif sequence (Supplementary Fig. 4a). Therefore, we performed chromatin immunoprecipitation (ChIP) using a PURB antibody and multiple $\mathrm{qPCR}$ primer pairs and showed that PURB has a high occupancy capacity over this region of Tns1. Importantly, the occupancy was impaired in MaTAR25 KO cells and was able to be restored upon ectopic expression of MaTAR25 in 4T1 MaTAR25 KO cells (Fig. 5e). Moreover, we found the binding ability of MaTAR25 to its downstream targeting regions (Tns1, PVT1, and Has2) was reduced in 4T1 PURB KO cells but not in PURA KO cells compared to 4T1 control cells using ChIRP-qPCR (Supplementary Fig. 5g). Together, these results provide compelling evidence indicating the interaction of PURB protein with MaTAR25 is required for PURB binding to regulatory motifs in the Tns 1 gene in $4 \mathrm{~T} 1$ cells.

Human IncRNA LINC01271 is the human ortholog of MaTAR25. In order to translate our exciting findings in regard to mouse MaTAR25 to the human system for potential future clinical applications, we went on to characterize the human ortholog of MaTAR25 and confirm its function in human breast cancer cells. Based on syntenic conservation between the human and mouse genomes, we previously found three lincRNAs as potential human counterparts of MaTAR25: LINC01270, LINC01271, and LINC0127230 (Fig. 6a). Among these three lncRNAs, only LINC01271 is transcribed in the same direction as MaTAR25. Analysis of The Cancer Genome Atlas (TCGA) data ${ }^{30}$ suggests two of these potential orthologs, LINC01270 and LINC01271, are expressed at increased levels in multiple subtypes of breast cancer (Fig. 6a). Therefore, we focused on these two lincRNAs and performed independent ectopic expression of LINC01270 and LINC01271 in 4T1 MaTAR25 KO cells to determine if one of these human lincRNAs could rescue the mouse MaTAR25 KO phenotype. Cell viability assays indicated that ectopic expression of LINC01271, but not LINC01270, can rescue the proliferation phenotype of MaTAR25 KO cells (Fig. 6b). Invasion assays also showed that ectopic expression of LINC01271 in 4T1 MaTAR25 KO cells can rescue the cell invasion phenotype (Fig. 6c). In addition, the expression of Tns 1 can also be restored to a similar level as in control 4T1 cells upon overexpression of LINC01271 in MaTAR25 KO cells (Fig. 6d). Immunoprecipitation (RIP) using the PURB antibody indicated a specific interaction between PURB and LINC01271 (Supplementary Fig. 6a) in human triple-negative breast cancer MDAMB-231 LM2 cells ${ }^{45}$. Next, we performed smRNA-FISH to examine the localization of LINC01271 within MDA-MB-231 LM2 cells and we found that similar to MaTAR25 it is a nuclearenriched RNA (Supplementary Fig. 6b). Together, these results validate LINC01271 as the human ortholog of MaTAR25.

LINC01271 may play a role in human breast cancer progression and have diagnostic and/or therapeutic potential. We performed ASO-mediated KD of LINC01271 in MDA-MB-231 LM2 cells and selected the three most effective LINC01271 ASOs to assess a KD phenotype. After $96 \mathrm{~h}$ of ASO treatment to mediate KD of LINC01271 in MDA-MB-231 LM2 cells (Fig. 6e, left panel), all three independent ASOs decreased cell viability by $\sim 32 \%$ (Fig. 6e, right panel). The KD result supports a role for LINC01271 in human breast cancer progression.

By using reactome pathway analysis with LINC01271 expression based on breast cancer TCGA data, we found the top pathways are related to the cell cycle (Supplementary Fig. 6c). In addition, according to the lncRNA database TANRIC ${ }^{46}$, higher expression of LINC01271 is correlated with poor breast cancer patient survival (Supplementary Fig. 6d). qRT-PCR analysis of the expression level of LINC01271 in breast tumor organoids vs organoids derived from normal adjacent breast tissue showed a higher expression level of LINC01271 in tumor-derived organoids (Supplementary Fig. 6e).

Next, we performed smRNA-FISH to localize LINC01271 in patient breast tumor sections. We found that LINC01271 expression level was increased with increased breast tumor stage (Fig. 7a and Supplementary Fig. 7a), and we identified the presence of clonal and regional differential expression patterns in most of the breast tumor patient samples (Supplementary Fig. 7b). Most interestingly, lung and lymph node metastases exhibited higher expression of LINC01271 than primary tumors from the same patients (Fig. 7b and Supplementary Fig. 7c). Thus, together these findings from patient-derived samples support our hypothesis that LINC01271 is a potential therapeutic target to impact breast cancer progression and metastasis.

\section{Discussion}

We identified MaTAR25 as a lncRNA that is upregulated in HER2 +, luminal, and TNBC as compared to normal mammary epithelial cells. Genetic KO or ASO KD of MaTAR25 results in a reduction in cell proliferation, migration, and invasion (Fig. 7c). Injection of 4T1 MaTAR25 KO cells into the mouse mammary fat pad or tail vein results in smaller tumor growth and a significant reduction in lung metastases. The human ortholog of MaTAR25 was identified as LINC01271, and it is expressed in primary breast tumors and at even higher levels in lymph node and lung metastases. Increased expression of the human ortholog of MaTAR25 is associated with poor patient prognosis ${ }^{46}$.

In order to understand the molecular mechanism by which MaTAR25 imparts its function, we used ChIRP-seq and RNA-seq to identify and validate the Tns1 gene as a direct downstream target of MaTAR25. MaTAR25 positively regulates the expression of Tns1 in mammary cancer cells through binding to its DNA sequence in trans. Tns1 has been shown to localize to focal adhesions and fibrilla adhesions, and to assist in mediating signaling between the extracellular matrix and the actin cytoskeleton to impact cell movement and proliferation ${ }^{36,37}$. Several reports have shown that loss of Tns 1 can cause a decrease in cell motility in many cell types ${ }^{47-49}$, and Tns 1 has also been shown to be involved in epithelial-mesenchymal transition (EMT) of cancer cells $^{50}$. The general relationship between the expression of Tns1 and different stages/subtypes of breast cancer has been unclear. However, Kaplan-Meier survival analysis indicates that the expression of Tns1 is increased in grade 3 breast tumors ${ }^{38}$ supporting our findings of a positive role of Tns1 in breast tumor progression mediated by MaTAR25. Although Tns1 is the top target of MaTAR25 with the highest statistical significance we cannot rule out the possibility that MaTAR25 also regulates other genes as well given the additional candidates identified in our ChIRP-seq analysis. These candidates will be the focus of future studies.

By performing RNA antisense oligonucleotide pulldown (RAP) in 4T1 cells combined with iTRAQ mass spectrometry, to determine the relative quantitation of MaTAR25-associated 
a

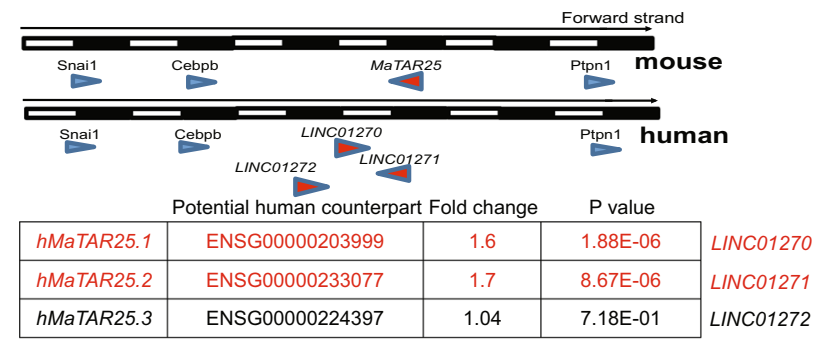

b

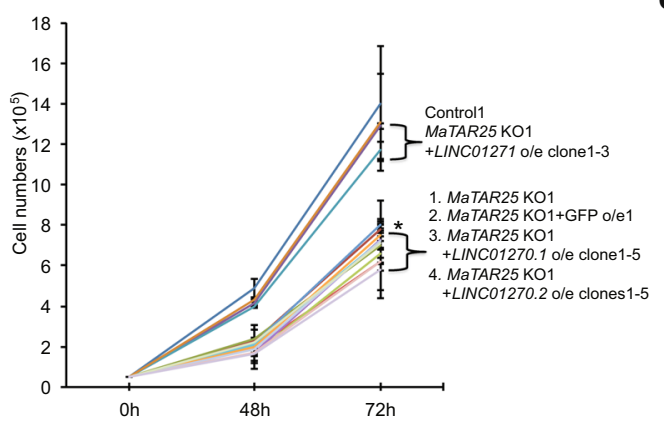

C
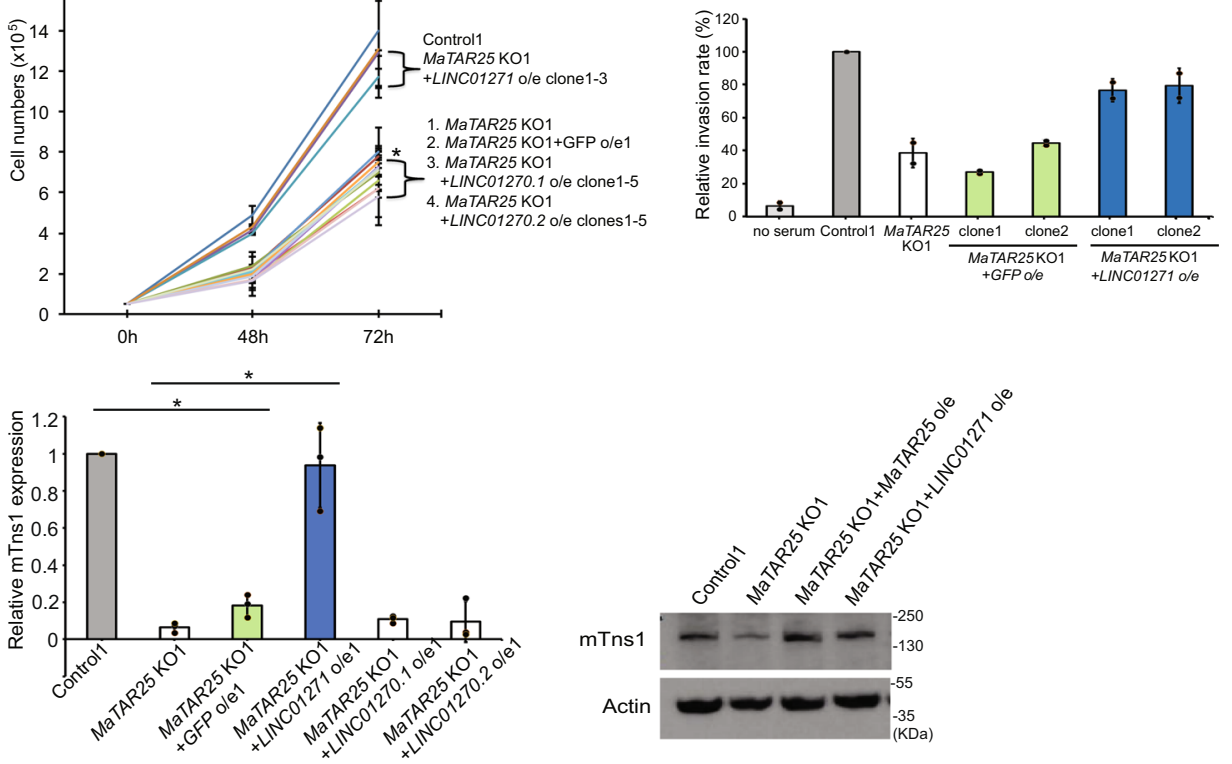

e
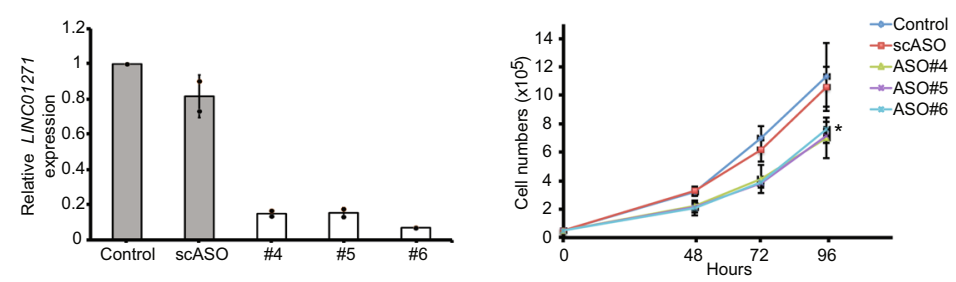

Fig. 6 Human LINC01271 is the human ortholog of MaTAR25. a All potential human orthologs of MaTAR25 (hMaTAR25) were identified based on conservation of genomic location (synteny). RNA-seq data from The Cancer Genome Atlas (TCGA) was analyzed to evaluate the expression status of all potential hMaTAR25 candidates by comparison of 1128 TCGA breast tumor datasets to 113 normal breast tissue controls. Fold change and statistical significance were calculated using the Wilcoxon rank test; $P$ values were adjusted using the Benjamini \& Hochberg method by DESeq2 ${ }^{30}$. b Attempted rescue of $4 T 1$ MaTAR25 KO cells upon ectopic expression of two transcript isoforms of LINC01270 (LINC01270.1, and LINC01270.2), or LINC01271 in cell viability assays. The mean cell numbers of three independent replicates of 4T1 control1, MaTAR25 KO1, MaTAR25 KO1 with GFP, LINC01270.1 clone1-5, LINC01270.2 clone1-5, and LINC01271 clone1-3 are shown \pm SD $(n=3){ }^{*} P<0.05$ (paired Student's $t$ test; two-tailed). c 4T1 MaTAR25 KO cells with ectopic expression of GFP was used as a control to assess rescue in a cell invasion assay. The mean relative cell invasion of two independent replicates of 4 T1 control, MaTAR25 KO1, MaTAR25 KO1 with GFP clone1-2, LINC01271 ectopic expression clone1-2 is shown \pm SD ( $n=2$ independent experiments). Ectopic expression of $L I N C 01271$ can rescue the MaTAR25 KO cell invasion phenotype. d RNA expression level of Tns1 was determined in MaTAR25 KO1 cells ectopically expressing LINC01270.1, LINC01270.2, or LINC01271 by qRT-PCR. Data are presented as mean values \pm SD ( $n=3$ independent experiments). ${ }^{\star} P<0.05$ (paired Student's $t$ test; two-tailed). The protein level of mTns1 was also examined in MaTAR25 KO cells with ectopic expression of LINCO1271 by immunoblot analysis. e Three different ASOs targeting LINC01271 were used to independently knockdown LINC01271 in MDA-MB-231 LM2 cells. Left panel: the knockdown efficiency is calculated and data are shown as mean values \pm SD ( $n=2$ independent experiments) by qRT-PCR after $24 \mathrm{~h}$ treatment of ASOs. Right panel: the mean cell numbers of three independent cell counting experiments of MDA-MB-231 LM2 mock-treated control cells, cells treated with scrambled ASO, and cells treated with three different LINCO1271 ASOs are shown $\pm \operatorname{SD}(n=3)$. ${ }^{\star} P<0.05$ (paired Student's $t$ test; two-tailed).

proteins, we identified a specific interaction between MaTAR25 and purine-rich element-binding protein B (PURB) which appears to be crucial for the downstream regulation of MaTAR25. PURB has been reported to be a transcriptional co-activator and binds to the single strand of the repeated purine-rich element PUR which is present in promoter regions and as such has been implicated in transcriptional control. PURB plays different roles in many physiological and pathological processes ${ }^{51-53}$. For 
a
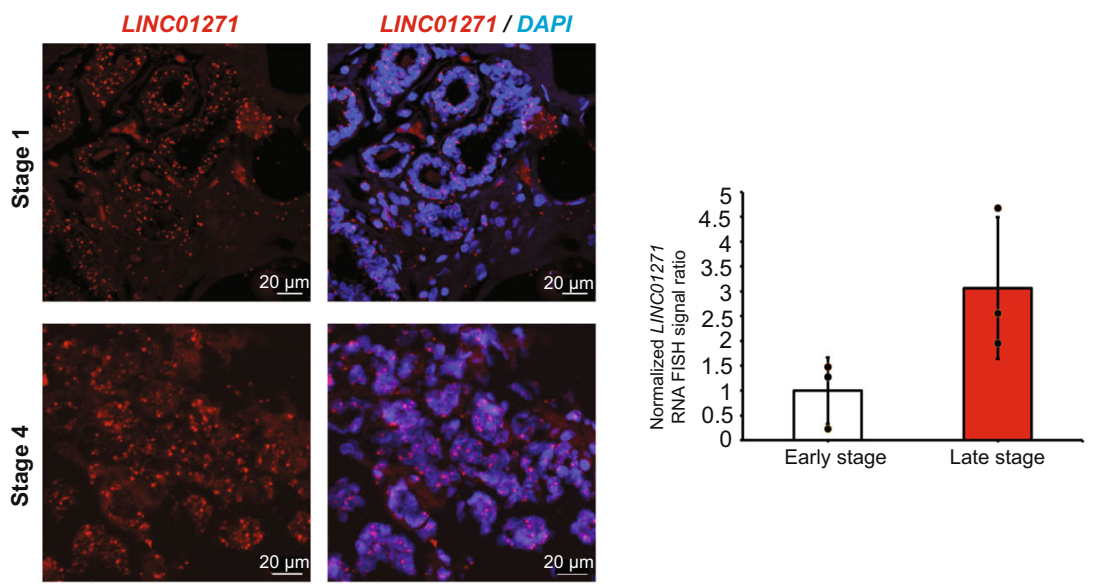

b

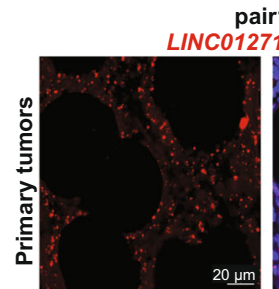

271 / DAPI
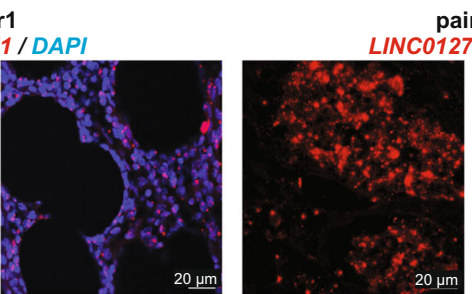

pair2 71 / DAPI
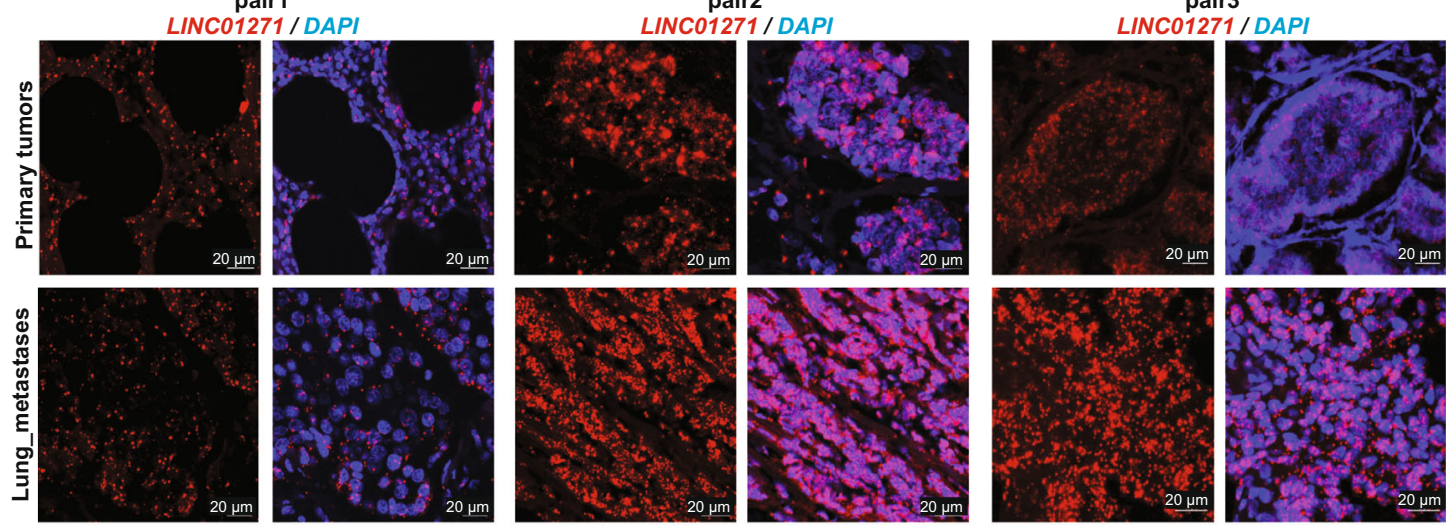

C

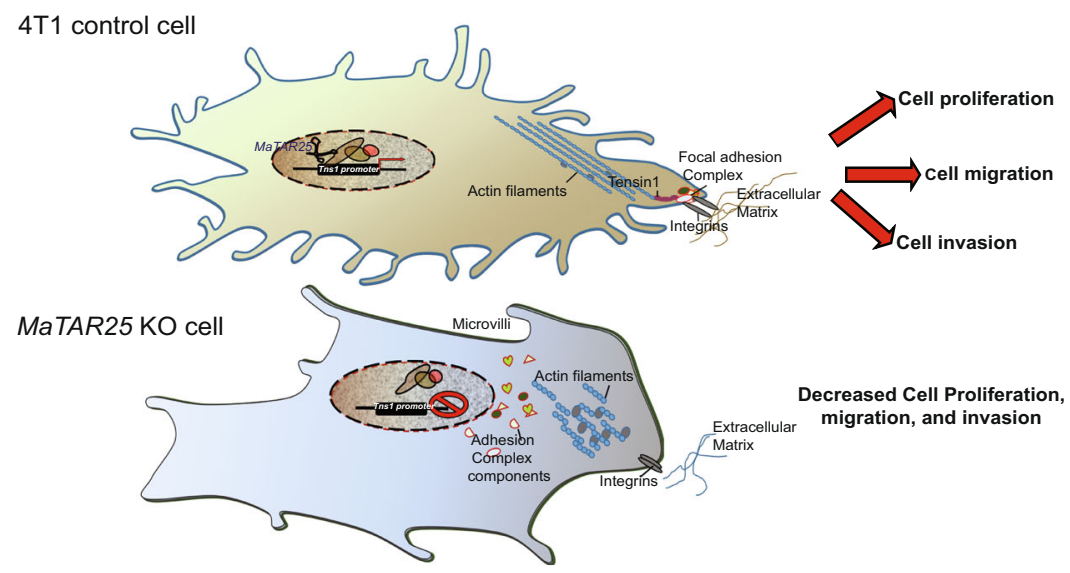

Fig. 7 LINC01271 expression in breast tumors and lung metastases. a smRNA-FISH images showing the expression of LINC01271 (red) in patient breast tumor sections from different stages of breast cancer. More than six random areas on each slide were examined, and representative images are shown. Scale bars are $20 \mu \mathrm{m}$. The quantification of six images (three early stage and three late stage) of breast tumor sections is shown and data are presented as mean values \pm SD. $P=0.08587$ (paired Student's $t$ test; two-tailed). $\mathbf{b}$ smRNA-FISH images showing the expression pattern of LINC01271 (red) within luminal subtype patient breast cancer primary tumors and lung metastases sections from the same patients. More than six random areas on each slide were examined, and representative images are shown. Scale bars are $20 \mu \mathrm{m}$. c Proposed model of MaTAR25 function. MaTAR25 acts as a scaffold and/or chaperone recruiting PURB to the Tns1 gene where it induces its transcription. The Tns1 protein associates with focal adhesion complexes regulating signaling between the extracellular matrix and the actin cytoskeleton. KO or KD of MaTAR25 results in a reorganization of the actin cytoskeleton and focal adhesion complexes as well as a significant decrease in microvilli resulting in a reduction in cell proliferation and migration. 
example, PURB has been shown to be overexpressed in several different cancer types ${ }^{54}$. In addition, a previous study showed PURB to act as a transcriptional co-factor that can be recruited by linc-HOXA1 to mediate the transcriptional regulation of lincHOXA1 in embryonic stem cells ${ }^{44}$ supporting a critical role of PURB with different lincRNAs in different biological contexts. We identified the MaTAR25 target region of the Tns1 gene to have a high ratio of purine to pyrimidine bases (3:1), and our ChIP-qPCR result indicated a high occupancy capacity of PURB over this targeting region, further supporting a functional role of PURB in partnering with MaTAR25 to regulate the Tns1 gene. The results of ectopic overexpression or siRNA-mediated knockdown of PURB, resulting in altered expression (upregulation or downregulation, respectively) of Tns1 in 4T1 mammary cancer cells indicating a transcriptional regulatory role of PURB in this context. As the interaction of PURB with MaTAR25 is essential for PURB binding to Tns1 DNA this suggests that MaTAR25 acts as a chaperone and/or scaffold for the MaTAR25/ PURB/Tns1 DNA complex, which is critical for transcriptional regulation of Tns1 thereby impacting cancer progression.

PURB can form a homodimer, a heterodimer with PURA, or a heterotrimer with PURA and Y-box protein 1 (YBX1). Interestingly, these two additional proteins were also identified in our MS-iTRAQ analysis of MaTAR25 interactors, and have been studied in many cancer types ${ }^{55-57}$. Future investigation of these potential interactions may provide further insights into the molecular mechanism of the MaTAR25- PURB complex in cancer cells.

Based upon synteny and further validation, we identified LINC01271 as the human ortholog of MaTAR25. Interestingly, LINC01271 has been identified as one of 65 new genetic loci that are related to overall breast cancer risk ${ }^{58}$. LINC01271 expression is increased in breast cancer and correlates with poor clinical outcome based on the analysis of patient clinical data ${ }^{46}$. In addition, our results from examining sections of breast tumors showed a positive correlation between the expression level of LINC01271 and the breast cancer stage. Our finding of an even higher level of expression in lung metastases from the same patients was especially interesting. Metastasis is the major cause of cancer-related deaths, particularly in breast cancer patients ${ }^{59,60}$, and developing an efficient treatment strategy to target and reduce breast cancer metastasis still remains the key challenge for this disease. Our ability to use ASOs to knockdown MaTAR25 in the HER2/neu mouse model resulting in necrotic tumors and a significant reduction in metastasis has therapeutic implications. Using ASO targeting as a therapeutic approach has been applied to many diseases ${ }^{61-63}$ including cancer ${ }^{26,64,65}$ in recent years. A cEt ASO targeting the transcription factor STAT3 has shown robust single-agent activity in highly treatmentrefractory lymphoma and non-small cell lung cancer studies ${ }^{64}$. The STAT3 ASO (AZD9150) has advanced into multiple Phase I and II clinical trials (NCT01563302, NCT02983578, NCT02549651). In addition, an antisense drug targeting all forms of the androgen receptor for the treatment of advanced metastatic prostate cancer has entered a clinical trial (NCT02144051). In this study, we developed three ASOs targeting LINC01271 for functional assays in vitro. Ultimately, LINC01271 is an ideal candidate to be exploited as a potential prognostic/therapeutic target and LINC01271-specific ASOs as therapeutics to impact breast cancer progression and metastasis.

\section{Methods}

Cell culture. Murine 4T1 cells, murine NF639 (cNeu; MMTV-Neu-NDL) cells, human MDA-MB-231 cells, and human MDA-MB-231 LM2 cells were cultured in DMEM with $10 \% \mathrm{FBS}$, and $1 \%$ penicillin/streptomycin. All cell lines were cultured in a cell culture incubator at $5 \% \mathrm{CO}_{2}$ at $37^{\circ} \mathrm{C}$.
Organoid culture. Surgically removed tumor samples from breast cancer patients along with adjacent normal tissue were collected from Northwell Health in accordance with Institutional Review Board protocol IRB-03-012 (TAP16-08). Informed consent ensured that the de-identified materials collected, the models created, and data generated from them can be shared without exceptions with researchers in the scientific community. Tumor and normal organoids were developed using a previously published protocol ${ }^{66}$. The tissues were manually cut into smaller pieces and treated with Collagenase IV (Sigma-Aldrich, C5138) at $37^{\circ} \mathrm{C}$. The samples were manually broken down by pipetting into smaller fragments and seeded in a dome of matrigel (Corning, 356231). Organoids were grown in culture media which contained $10 \%$ R-Spondin 1 conditioned media, $5 \mathrm{nM}$ neuregulin 1 (Peprotech, 100-03), $5 \mathrm{ng} / \mathrm{ml}$ FGF7 (Peprotech, 100-19), $20 \mathrm{ng} / \mathrm{ml}$ FGF10 (Peprotech, 100-26), $5 \mathrm{ng} / \mathrm{ml}$ EGF (Peprotech, AF-100-15), $100 \mathrm{ng} / \mathrm{ml}$ noggin (Peprotech, 120-10 C), $500 \mathrm{nM}$ A83-01 (Tocris, 2939), $5 \mu \mathrm{M} \mathrm{Y-27632}$ (Abmole, Y-27632), 1.2 $\mu$ M SB202190 (Sigma-Aldrich, S7067), 1× B27 supplement (Gibco, 17504-44), $1.25 \mathrm{mM} \mathrm{N}$-acetylcysteine (Sigma-Aldrich, A9165), $5 \mathrm{mM}$ nicotinamide (Sigma-Aldrich, N0636), $1 \times$ glutamax (Invitrogen, 12634-034), $10 \mathrm{mM}$ HEPES (Invitrogen, 15630-056), $100 \mathrm{U} / \mathrm{ml}$ Pen-Strep (Invitrogen, 15140122) $50 \mu \mathrm{g} / \mathrm{ml}$ Primocin (Invitrogen, ant-pm-1) in 1× advanced DMEM-F12 (Invitrogen, 12634-034) ${ }^{66}$. Cultures were passaged every $2-4$ weeks using TrypLETM (Thermo Fisher, 12605028) to break down the organoids into smaller clusters of cells and re-plating them in matrigel.

Organoid RNA extraction. RNA was extracted using TRIzol ${ }^{\circ}$ (Thermo Fisher, 15596018) RNA extraction protocol followed by suspension in nuclease-free deionized water. Reagents used in this study are provided in Supplementary Data 6

Mice. All animal procedures and studies were approved by the Cold Spring Harbor Laboratory Animal Use Committee in accordance with IACUC procedures. Mice were maintained, and the experiments were performed with oversight of the Cold Spring Harbor Laboratory Animal Shared Resource, which is fully accredited by the Association for Assessment and Accreditation of Laboratory Animal Care (AAALAC). Briefly, male MMTV-Neu-NDL mice (FVB/N background) were kindly provided by Dr. William Muller (McGill University, Canada). Male MMTV-NeuNDL mice were crossed with wild type FVB/N female mice purchased from The Jackson Laboratory for breeding. PCR genotyping was applied to select female mice with heterogeneous genotypes for MMTV-Neu-NDL for later in vivo ASO injection experiments. In all, 4-6-week-old $\mathrm{BALB} / \mathrm{c}$ female mice were purchased from the Jackson Laboratory for in vivo 4T1 cell mammary fat pad (MFP) injection and tail-vein injection experiments.

$\mathbf{5}^{\prime} / \mathbf{3}^{\prime}$ rapid amplification of cDNA ends (RACE). $5^{\prime}$ and $3^{\prime}$ RACE of MaTAR25 transcripts was performed on TRIzol-extracted RNA using the Invitrogen FirstChoice RLM-RACE kit (Thermo Fisher, AM1700M) according to the manufacturer's instructions. Briefly, fragments were amplified by nested PCR using AmpliTaq Polymerase. PCR products were separated on 2\% agarose, bands excised, gel purified, sub-cloned into pGEM-T Easy vector (Promega, A137A), and four or more clones per fragment were sequenced using standard Sanger sequencing. Primer sequences are provided in Supplementary Data 8.

Northern blot analysis. The total RNA was isolated from MMTV-PyMT tumor cells using the TRIzol method, and the quality of the RNA sample (A260/A280 ratio) was measured using a Nanodrop 2000 (Thermo Fisher Scientific). MaTAR25-specific radiolabeled DNA probes were generated using dCTP ${ }^{32} \mathrm{P}$ (PerkinElmer, BLU513H250UC) in a random primed labeling reaction. The total RNA was extracted by the TRIzol method. Analysis of RNA expression was performed by following the NorthernMax Kit manual (Thermo Fisher, AM8671, AM8676, and AM8677). Briefly, $20 \mu \mathrm{g}$ and $30 \mu \mathrm{g}$ of total RNA samples were denatured at $70^{\circ} \mathrm{C}$ for $10 \mathrm{~min}$ and electrophoresed in a $1 \%$ agarose gel $(\sim 4.5 \mathrm{~h})$. The separated RNA was transferred from the gel to a positively charged nylon membrane (NC) by capillary blotting at room temperature overnight (or $16 \mathrm{~h}$ ). The transferred RNA was then fixed to the NC membrane using UV cross-linking by Stratalinker on automode. The cross-linked membrane was prehybridized with ultrahyb-oligo hybridization buffer (Thermo Fisher, AM8663) and hybridized with the heat-denatured MaTAR25-specific radiolabeled DNA probe in a roller hybridization bottle at $42^{\circ} \mathrm{C}$ overnight. After washing with SSC (G-Biosciences, R020) wash buffer by the steps: $2 \times$ SSC with $1 \%$ SDS at $42^{\circ} \mathrm{C}$ twice for 10 min each time, $1 \times$ SSC with $0.5 \%$ SDS at $42{ }^{\circ} \mathrm{C}$ twice for 10 min each time, and $1 \times$ SSC with $0.1 \%$ SDS at $37^{\circ} \mathrm{C}$ twice for $10 \mathrm{~min}$ each time, the wrapped membrane was exposed to a phosphorImager screen in a cassette or X-ray film (VWR, 95017-661) for signal detection. For stripping the membrane, the membrane was incubated in $50 \mathrm{ml} 0.1 \%$ SDS solution (just to boiling; $\sim 4 \mathrm{~min}$ in the microwave) in the roller hybridization bottle for $15 \mathrm{~min}$. The SDS solution was discarded in a radioactive waste bottle. The steps were repeated again (total two washes), and the membrane was checked with a Geiger counter before re-probing. Reagents used in this study are provided in Supplementary Data 6.

Cell lysate preparation for immunoblot analysis. Cells were trypsinized, and harvested cell pellets were lysed in RIPA buffer (Thermo Scientific, 89900; $25 \mathrm{mM}$ 
Tris- $\mathrm{HCl} \mathrm{pH} 7.6,150 \mathrm{mM} \mathrm{NaCl}, 1 \% \mathrm{NP}-40$ substitute, $1 \%$ sodium deoxycholate, and $0.1 \%$ SDS) supplemented with $1 \times$ Roche protease inhibitor cocktail. The cell lysate was incubated on ice for $15 \mathrm{~min}$, then sonicated for $5 \mathrm{~min}$ before centrifugation at 13,000 $\times g$. The supernatant was collected and quantified using the Pierce $^{\text {tw }}$ BCA protein assay kit (Thermo Fisher, 23225).

In vitro transcription/translation. T7 promoter containing DNA sequences or plasmids were used in a TNT ${ }^{\oplus}$ Quick Coupled Transcription/Translation System (Promega, L1170) following the manufacture's protocol. Briefly, $1 \mu \mathrm{g}$ plasmid DNA template was mixed with $40 \mu \mathrm{l}$ TNT $^{\oplus}$ T7 Quick Master Mix, $1 \mu$ l methionine (1 $\mathrm{mM}), 1 \mu \mathrm{l}$ Transcend ${ }^{\mathrm{m}}$ Biotin-Lysyl-tRNA, and nuclease-free water for the final volume of $50 \mu \mathrm{l}$ per reaction. The reaction tube was incubated at $30^{\circ} \mathrm{C}$ for $90 \mathrm{~min}$, and $1 \mu \mathrm{l}$ reaction product was added into diluted $2 \times$ Laemmli sample buffer (BIORAD, 1610737) for immunoblot analysis. Samples were loaded on $4-20 \%$ MiniPROTEAN $^{\circledR}$ TGX $^{\mathrm{m}}$ Precast Protein Gels (BIO-RAD, 4561094), and the signals were detected by streptavidin-HRP (Jackson Immunoresearch, 016-030-084).

\section{RNA isolation and quantitative real-time PCR (qRT-PCR) assays. The total} RNA was extracted using TRIzol following the manufacture's protocol (* make sure to use nuclease-free reagents and tools). In general, $1 \mathrm{ml}$ TRIzol was added for the RNA extraction to a $1.5-\mathrm{ml}$ tube containing the cell pellet $\left(5-10 \times 10^{6}\right)$ in the chemical hood. The sample was completely dissociated in the TRIzol reagent at room temperature for $5 \mathrm{~min}$ for homogenization. In all, $200 \mu \mathrm{l}$ of chloroform per 1 $\mathrm{ml}$ of TRIzol reagent was added into the sample tube, and the sample tube was inverted ten times then incubated at room temperature for $3 \mathrm{~min}$. The sample was centrifuged at $12,000 \times g$ for $15 \mathrm{~min}$ at $4{ }^{\circ} \mathrm{C}$ for phase separation. The colorless upper aqueous phase containing RNA was transferred carefully to the new $1.5-\mathrm{ml}$ tube, and $0.5 \mathrm{ml}$ of isopropyl alcohol per $1 \mathrm{ml}$ of TRIzol reagent and $1 \mu \mathrm{l} \mathrm{Glyco-}$ Blue $^{\text {mo }}$ Coprecipitant (Thermo Fisher, AM9516) were added. The sample tube was inverted ten times and incubated at room temperature for $10 \mathrm{~min}$ or $-20^{\circ} \mathrm{C}$ overnight for RNA precipitation. The sample was centrifuged at $12,000 \times g$ for 15 min at $4{ }^{\circ} \mathrm{C}$. The blue color RNA pellet was visible on the bottom of the tube after centrifugation. After removing the supernatant, $1 \mathrm{ml}$ of $75 \%$ ethanol per $1 \mathrm{ml}$ of TRIzol reagent was added to the sample tube to wash the RNA pellet. The sample tube was vortexed for $10 \mathrm{~s}$ and centrifuged at $10,000 \times g$ for $10 \mathrm{~min}$ at $4^{\circ} \mathrm{C}$. After centrifugation, the ethanol was removed completely and the RNA pellet was airdried at room temperature but not dried completely $(\sim 10 \mathrm{~min})$. The dried RNA pellet was dissolved in 10-20 $\mu$ Invitrogen nuclease-free water (Thermo Fisher, AM9932) and incubated at $50^{\circ} \mathrm{C}$ in a shaking incubator for $10 \mathrm{~min}$. The quality and quantity of the RNA sample were measured using a Nanodrop 2000 (Thermo Fisher Scientific) to check A260/A230 and A260/A280 ratios. RNA samples with 1.8-2.0 A260/A280 ratios were used as high-quality samples. In all, $1 \mu \mathrm{g}$ of the total RNA was treated with DNaseI (Thermo Fisher, 18068015) and reverse transcribed into cDNA using TaqMan Reverse Transcription Reagent kit (Thermo Fisher, 4304134), followed by qPCR with SYBR green PCR master mix (Thermo Fisher, A25743) on an ABI QuantStudio 6 Flex Real-Time PCR System. qRT-PCR conditions were as follows: $30 \mathrm{~min}$ at $50^{\circ} \mathrm{C}$ for reverse transcription, $15 \mathrm{~min}$ at $95^{\circ} \mathrm{C}$ for the initial activation step followed by 40 cycles of $15 \mathrm{~s}$ at $94^{\circ} \mathrm{C}, 30 \mathrm{~s}$ at $60^{\circ} \mathrm{C}$. Mouse peptidylprolyl isomerase B (cyclophilin B; Ppib) and human GAPDH and RPL13A were used as endogenous controls to normalize each sample. Reagents used in this study are provided in Supplementary Data 6, and primer sequences are provided in Supplementary Data 8.

\section{Cell fractionation, cytoplasmic/nucleoplasmic/chromatin-related RNA isola-} tion. Cell fractionation was done using a standardized protocol ${ }^{67}$. After reaching $80 \%$ confluence, cultured $4 \mathrm{~T} 1$ cells were trypsinized and counted. In total, $3 \times 10^{6}$ $4 \mathrm{~T} 1$ cells were collected and lysed in NP-40 substitute (Thermo Fisher, AAJ19628AP) lysis buffer (10 mM Tris pH 7.5, $150 \mathrm{mM} \mathrm{NaCl}$, and 0.15\% NP-40 substitute) supplemented with SUPERase-In RNase Inhibitor (Thermo Fisher, AM2696) on ice for $5 \mathrm{~min}$. Ten percent of the cell lysate was collected into a new 1.5 -ml tube on ice for RNA extraction later as the reference input. Ninety percent of the cell lysate was overlaid on top of sucrose buffer $(10 \mathrm{mM}$ Tris pH 7.4, $150 \mathrm{mM}$ $\mathrm{NaCl}$, and $24 \%$ sucrose) and centrifuged at $3500 \times g$ for $10 \mathrm{~min}$ to separate the cytoplasmic fraction and nuclei. The supernatant was transferred to a new $1.5-\mathrm{ml}$ tube as the cytoplasmic fraction, and the pellet as the nuclei. Next, the nuclear pellet was rinsed with PBS-EDTA once and resuspended with glycerol buffer $(20$ $\mathrm{mM}$ Tris $\mathrm{pH}$ 7.4, $75 \mathrm{mM} \mathrm{NaCl}, 0.5 \mathrm{mM}$ EDTA, and $50 \%$ glycerol) supplemented with SUPERase-In RNase Inhibitor mixed with urea buffer (1 M Urea, $0.3 \mathrm{M} \mathrm{NaCl}$, $7.5 \mathrm{mM} \mathrm{MgCl}, 0.2 \mathrm{mM}$ EDTA, and 1\% NP-40 substitute) on ice for $2 \mathrm{~min}$. The lysate was then centrifuged at $13,000 \times g$ for 2 min to separate the nucleoplasmic fraction and chromatin pellet. The chromatin was resuspended in TRIzol reagent and fully solubilized by passing through the 21-gauge needle/syringe. The cytoplasmic fraction and nucleoplasmic fractions were also used for RNA extraction using TRIzol reagent. RNA extracted from different fractions was applied for cDNA synthesis and qRT-PCR. Primer sequences are provided in Supplementary Data 8.

CRISPR/Cas9 genetic knockout. To generate a genetic knockout of MaTAR25, two sgRNAs targeting the promoter region were combined, creating a deletion including the TSS. Both sgRNAs were designed using http://crispr.mit.edu/. The sgRNA targeting the gene body of MaTAR25 was cloned into a pSpCas9(BB)-2AGFP vector (Addgene, 48138), and the sgRNA targeting the upstream promoter region was cloned into a pSpCas9(BB)- 2A-mCherry vector. $4 \mathrm{~T} 1$ cells were transfected with both plasmids using SE Cell Line 4D-Nucleofector kit (Lonza, V4XC-1024) in 4D-Nucleofector X Unit (Lonza) with program code "CN-114". Cells transfected with a sgRNA targeting Renilla luciferase were used as a negative control. To select for cells expressing both gRNAs, GFP and mCherry doublepositive cells were sorted 40-h post transfection, as single-cell deposition into 96well plates using a FACS Aria (SORP) Cell Sorter (BD) (Supplementary Data 10). Each single-cell clone (control and MaTAR25 KO) was propagated and analyzed by genomic PCR and qRT-PCR to select for homozygous knockout clones.

To generate a genetic knockout of Tns1, PURB, and PURA, sgRNAs targeting different exons of genes were designed using http://crispr.mit.edu/. The sgRNAs were cloned into a pSpCas9(BB)-2A-Puro (PX459) V2.0 vector (Addgene, 62988). $4 \mathrm{~T} 1$ cells were transfected using SE Cell Line 4D-Nucleofector kit (Lonza, V4XC$1024)$ in 4 D-Nucleofector X Unit (Lonza) with program code "CN-114". Cells transfected with a sgRNA targeting Renilla luciferase were used as a negative control. Transfected cells were placed under $2 \mu \mathrm{g} / \mathrm{ml}$ puromycin (Sigma-Aldrich, P8833) in culture media for selection. Selected pool cells were propagated and analyzed by qRT-PCR and western blotting to confirm. Assays and resources used in this study are provided in Supplementary Data 7, and sequences for all sgRNAs and primers are provided in Supplementary Data 8.

Cell counting viability assay. After reaching $80 \%$ confluence in a 100 -mm culture dish, cells were trypsinized and harvested. Cells were resuspended and applied to a hemocytometer for manual counting. The same number of cells $\left(2.5\right.$ or $\left.5 \times 10^{4}\right)$ of each group was seeded into each well of a six-well tissue culture plate at day 0 and then seeded cells were incubated in a $37^{\circ} \mathrm{C}$ incubator with $5 \% \mathrm{CO}_{2}$. At different time points of incubation, cells were trypsinized and harvested for manual counting. Trypan Blue-treated cell suspensions were collected and applied to a hemocytometer for manual counting.

Cell cycle analysis. Cell cycle analysis was performed using BD bromodeoxyuridine (BrdU) FITC assay kit (BD, 559619) following the manufacturer's protocol. Briefly, cultured cells were incubated with BrdU containing medium for $30 \mathrm{~min}$, and FITC-conjugated anti-BrdU antibody was applied for labeling actively synthesizing DNA. 7-aminoactinomycin D (7-AAD) was used for labeling total DNA. The labeled cell samples were analyzed on the BD LSR II flow cytometer, and BrdU FITC-A vs DNA 7-AAD dot plot with gates was used to encompass the G0/G1, S and G2/M populations. The collected cytometry data were analyzed with FACSDiva ${ }^{\text {mat }}$ and FlowJo software.

Migration assay. Live-cell tracking was performed to examine cell migration over time. After reaching $80 \%$ confluence on $100-\mathrm{mm}$ culture dish, cells (4T1 control clones and 4T1 MaTAR25 KO clones) were harvested and the same number of cells $\left(2 \times 10^{4}\right)$ were seeded into six-well tissue culture plates at day 0 . Cells were cultured in a $37^{\circ} \mathrm{C}$ cell culture incubator at $5 \% \mathrm{CO}_{2}$ for $12 \mathrm{~h}$ before imaging.

Cells were imaged in the microscope chamber at $37^{\circ} \mathrm{C}$ with $5 \% \mathrm{CO}_{2}$, and images were collected every $5 \mathrm{~min}$ (five viewpoints were selected from each well) using a Zeiss AxioObserver microscope for $8 \mathrm{~h}$. The images of the individual samples were converted to videos using ImageJ. Videos were analyzed by CellTracker image-processing software (https://academic.oup.com/bioinformatics/ article/32/6/955/1744578), and the migration distance ( $\mu \mathrm{m}$ or pixels) of cells were measured by manual and semi-automatic tracking. The mean relative migration distance ( $\mu \mathrm{m}$ or pixels) of three independent replicates of $4 \mathrm{~T} 1$ control clone groups and MaTAR25 KO clone groups were calculated.

Scratch wound-healing assay. Cultured cells were harvested and seeded into each well of a 24-well tissue culture plate. Cells were incubated until they reached at least $90 \%$ confluence. The wound line was created by "scratch" with a p200 micropipette tip, and cells were washed with PBS twice to remove the debris and smooth the edge of the scratch. To create markings close to the wound line as reference points for imaging, we labeled the outer bottom of the dish with a marker pen. At least two areas of each well were imaged immediately as the time point $0 \mathrm{~h}$, and culture plates were placed back in the incubator at $37^{\circ} \mathrm{C}$ for $8-24 \mathrm{~h}$. During the incubation, the plates were brought out for imaging every $6-8 \mathrm{~h}$ and returned to the incubator to determine the actual endpoint for specific cell types. For $4 \mathrm{~T} 1$ cells, the endpoint of the scratch wound-healing assay is $12 \mathrm{~h}$. After $12 \mathrm{~h}$ of incubation, the same areas based on the reference points of each well were imaged again, and the migration areas in each well were measured by ImageJ for comparison.

Invasion assay. Invasion assays with $4 \mathrm{~T} 1 \mathrm{MaTAR} 25$ knockout cells and $4 \mathrm{~T} 1$ control cells were carried out using the Cultrex 24 -well BME Cell Invasion Assay (Trevigen, 3455-024-K) following the manufacturer's protocol. BME coating: The day before the experiment, the inserts and culture well plates were assembled as assay chambers, and $100 \mu \mathrm{l}$ of $0.1-1 \times$ BME stock solution was prepared as the coating solution (for $4 \mathrm{~T} 1$ cells, use $0.5 \times \mathrm{BME}$ stock solution) for each insert. Coated chambers were incubated at $37^{\circ} \mathrm{C}$ overnight. Starvation: After reaching 
$70 \%$ confluence in $100-\mathrm{mm}$ culture dish, cells were washed with PBS twice, and serum-free DMEM medium once. Cells were starved by incubating in serum-free DMEM medium for $12 \mathrm{~h}$ before the experiment. Incubation for cell invasion: Cells were harvested and resuspended using serum-free DMEM medium. After counting, cells were diluted and seeded at a density of $1 \times 10^{5}$ cells in $100 \mu$ serum-free DMEM medium per well into the invasion assay chamber. Just before seeding cells, the coating solution was carefully aspirated from the insert without touching the membrane. In all, $500 \mu \mathrm{l}$ of culture medium (DMEM with $10 \% \mathrm{FBS}$ ) was added to the bottom chambers, and serum-free DMEM medium was used that did not stimulate cell invasion through the BME as a negative control. The plate was incubated at $37^{\circ} \mathrm{C}$ for $24 \mathrm{~h}$, and the assay was performed (incubation times for the assay varied from $4 \mathrm{~h}$ to $48 \mathrm{~h}$ for different cell types, and needed to be determined before the actual experiment). Measurement of invaded cells: After $24 \mathrm{~h}$ of incubation, the upper medium was removed, and cells from the top of inserts were washed with $100 \mu \mathrm{l} 1 \times$ wash buffer without puncturing the membrane. The upper part of inserts was cleaned again using cotton tipped swabs gently without breaking the membrane. The insert was taken out, and the medium was aspirated from bottom chamber, and wash each well with $500 \mu \mathrm{l} 1 \times$ wash buffer. The wash buffer was aspirated; $500 \mu \mathrm{l}$ of Cell Dissociation Solution/Calcein-AM was added to the bottom chamber of each well. The inserts were added back to the plates, and the plates were incubated at $37^{\circ} \mathrm{C}$ in a $5 \% \mathrm{CO}_{2}$ incubator for $1 \mathrm{~h}$ (gently swirl the plates once during the incubation). After $1 \mathrm{~h}$ of incubation, the invaded $4 \mathrm{~T} 1$ cells through the BME layer and attached to the bottom of the invasion insert were collected using cell dissociation solution and stained with Calcein-AM as the assay solution. The upper insert was removed, and the fluorescence of the assay solution was measured with a SpectraMax i3 Multi-Mode Detection Platform (Molecular Devices) using the Ex480/Em520 nm filter set.

Cloning. Specific gene overexpression plasmids were constructed using NEBuilder HiFi DNA Assembly (NEB, E5520S) following the manufacture's protocol. PCR products were cloned into pCMV6-entry plasmids digested with Sgf I and Fse I. Assembled plasmids were introduced into NEB stable competent $E$. coli using heat shock transformation and kanamycin selection. Four or more colonies per plate were picked and sequenced using standard Sanger sequencing.

\section{Antisense oligonucleotide (ASO) and siRNA-mediated knockdown (KD).} Specific 16mer antisense oligonucleotides (ASOs) comprised of phosphorothioatemodified short S-cEt (S-2'-O-Et-2',4'-bridged nucleic acid) gapmer chemistry targeting MaTAR25 and LINC01271 were designed and provided by Ionis Pharmaceuticals, Inc. Briefly, cultured cells were harvested and seeded into culture dishes. Transfection-free uptake of ASOs was accomplished by adding $4 \mu \mathrm{M}$ of either MaTAR25/LINC01271-specific ASOs or scrambled ASO (scASO) to the culture medium immediately after seeding the cells. Cells were incubated for indicated time points, and RNA was isolated using TRIzol reagent for qRT-PCR to check the knockdown efficiency. For siRNA-mediated knockdown, siRNAs (27mers) targeting mouse PURB were purchased from ORIGENE (ORIGENE, SR410793), and siRNA transfection was done using Lipofectamine 2000 (Thermo Fisher, 11668030) following the manufacture's protocol. RNA was extracted at different time points for qRT-PCR to check the knockdown efficiency. ASO sequences and primer sequences are provided in Supplementary Data 8.

Chromatin isolation by RNA purification (ChIRP)-Seq. For Chromatin Isolation by RNA Purification (ChIRP), we followed a previously described protocol ${ }^{35}$. Briefly, 20 million cells were harvested and fixed in $1 \%$ glutaraldehyde solution (Sigma-Aldrich, G5882) for each reaction. ChIRP was performed using biotinylated oligo probes designed against mouse MaTAR25 using the ChIRP probe designer (Biosearch Technologies). Independent even and odd probe pools were used to ensure IncRNA-specific retrieval (refer to Supplementary Data 8 for odd and even sequences targeting MaTAR25, and probes targeting mouse Ppib transcripts which were used as negative controls). ChIRP-Seq libraries were constructed using the Illumina TruSeq ChIP Library Preparation Kit (Illumina, IP-202-1012). Sequencing libraries were barcoded using TruSeq adapters and sequenced on Illumina NextSeq instruments.

ChIRP-Seq data analysis. Data quality was assessed using FastQC (http://www. bioinformatics.babraham.ac.uk/projects/fastqc/) and paired-end reads were mapped to GRCm 38 using Bowtie ${ }^{68}$ with parameters --end-to-end --sensitive --fr, resulting in a $90 \%$ or higher overall alignment rate. ChIRP-seq analysis was performed using HOMER ${ }^{69}$. Differential ChIRP peaks were called using the getDifferentialPeaksReplicates.pl script, with negative control (Ppib) pull-down samples as background and parameters -style histone -f 50. Peaks identified with at least a 50 -fold enrichment were processed further using the annotatePeaks.pl script and the GENCODE vM16 annotation. Both known and de novo motif analysis were carried out with the findMotifsGenome.pl script on the repeat-masked GRCm38 genome, $+/-500$ bp around the identified ChIRP peaks.

RNA-seq library construction. RNA was extracted using TRIzol following the manufacture's protocol. RNA quality was assayed by running an RNA 6000 Nano chip (Agilent, 5067-1511) on the Agilent 2100 Bioanalyzer. In total, $1 \mu \mathrm{g}$ of the total
RNA was used for constructing each RNA-seq library using the Illumina TruSeq sample prep kit v2 (Illumina, RS-122-2001) following the manufacturer's protocol. Briefly, RNA was polyA selected and enzymatically fragmented. cDNA was synthesized using Super Script II master mix (Thermo Fisher, 18064014), followed by end repair, A-tailing, and PCR amplification. Each library was high-throughput single-end sequenced on Illumina NextSeq instruments.

RNA-Seq data analysis. Data were analyzed as previously described ${ }^{30}$. Briefly, the quality of FASTQ files was assessed using FastQC (http://www.bioinformatics. babraham.ac.uk/projects/fastqc/). Reads were mapped to GRCm38 using $\mathrm{STAR}^{70}$ and the reads per gene record were counted using HTSeq-count ${ }^{71}$ and the GENCODE vM5 annotation. Differential gene expression was performed with DESeq2 ${ }^{72}$, and a FDR-adjusted $P$ value of $<0.1$ was set as a threshold for statistical significance. KEGG pathway and GO term enrichment and was carried out using the R/Bioconductor packages GAGE ${ }^{73}$ and Pathview ${ }^{74}$.

RNA antisense pulldown for mass spectrometry. Cells were lysed in a $10-\mathrm{cm}$ culture dish in $1 \mathrm{ml}$ of IPLB, Pierce ${ }^{\mathrm{ms}}$ IP Lysis Buffer (Thermo Fisher, 87787; $25 \mathrm{mM}$ Tris- $\mathrm{HCl} \mathrm{pH} \mathrm{7.4,} 150 \mathrm{mM} \mathrm{NaCl}, 1 \% \mathrm{NP}-40,1 \mathrm{mM}$ EDTA, 5\% glycerol) supplemented with $100 \mathrm{U} / \mathrm{ml}$ SUPERase-IN (Thermo Fisher, AM2696) and 1× Roche protease inhibitor cocktail (Sigma-Aldrich, 11836170001) for $10 \mathrm{~min}$, and the lysate was centrifuged at $13,000 \times g$ for $10 \mathrm{~min}$. The cell lysate was adjusted to $0.3 \mathrm{mg} / \mathrm{ml}$ (Pierce ${ }^{\mathrm{m}}$ BCA Protein Assay). A total of $100 \mathrm{pmol}$ of biotinylated oligo was added to $500 \mu$ l of lysate and incubated at room temperature for $1 \mathrm{~h}$ with rotation. In all, $100 \mu \mathrm{l}$ of streptavidin Dynabeads were washed in IPLB, added to the lysate, and incubated for $30 \mathrm{~min}$ at room temperature with rotation. Beads were washed three times with $1 \mathrm{ml}$ lysis buffer. For determining temperature for optimal elution, beads were then resuspended in $240 \mu \mathrm{l}$ of $100 \mathrm{mM}$ TEAB and aliquoted into eight PCR tubes. The temperature was set on a veriflex PCR block and incubated for 10 min. Beads were captured, and TRIzol was added to the eluate and beads. Once the optimal temperature is established, the beads were resuspended in $90 \mu \mathrm{l}$ of $100 \mathrm{mM}$ $\mathrm{TEAB}$, and incubated at $50^{\circ} \mathrm{C}$ for $10 \mathrm{~min}$. TRIzol was added to $30 \mu \mathrm{l}$ of the eluate, another $30 \mu \mathrm{l}$ was kept for immunoblots, and the last $30 \mu \mathrm{l}$ aliquot was sent directly to the Cold Spring Harbor Laboratory Mass Spectrometry Shared Resource.

Mass spectrometry. Tryptic digestion and iTRAQ labeling-Eluted samples were reduced and alkylated with $5 \mathrm{mM}$ DTT and $10 \mathrm{mM}$ iodoacetamide for $30 \mathrm{~min}$ at $55^{\circ} \mathrm{C}$, then digested overnight at $37^{\circ} \mathrm{C}$ with $1 \mu \mathrm{g}$ Lys-C (Promega, VA1170) and dried in vacuo. Peptides were then reconstituted in $50 \mu \mathrm{l}$ of $0.5 \mathrm{M} \mathrm{TEAB} / 70 \%$ ethanol and labeled with 4-plex iTRAQ reagent for $1 \mathrm{~h}$ at room temperature essentially as previously described ${ }^{75}$. Labeled samples were then acidified to $<\mathrm{pH} 4$ using formic acid, combined and concentrated in vacuo until $\sim 10 \mu \mathrm{l}$ remained.

Two-dimensional fractionation-Peptides were fractionated using a Pierce ${ }^{\mathrm{wn}}$ High pH Reversed-Phase Peptide Fractionation Kit (Thermo Scientific, 84868) according to the manufacturer's instructions with slight modifications. Briefly, peptides were reconstituted in $150 \mu \mathrm{l}$ of $0.1 \%$ TFA, loaded onto the spin column, and centrifuged at $3000 \times g$ for $2 \mathrm{~min}$. Column was washed with water, and then peptides were eluted with the following percentages of acetonitrile (ACN) in $0.1 \%$ triethylamine (TEA): $5 \%, 7.5 \%, 10 \%, 12.5 \%, 15 \%, 20 \%, 30 \%$, and $50 \%$. Each of the 8 fractions was then separately injected into the mass spectrometer using capillary reverse-phase LC at low $\mathrm{pH}$.

Mass spectrometry-An Orbitrap Fusion Lumos mass spectrometer (Thermo Scientific), equipped with a nano-ion spray source was coupled to an EASY-nLC 1200 system (Thermo Scientific). The LC system was configured with a self-pack PicoFrit ${ }^{\mathrm{n}}$ 75- $\mu \mathrm{m}$ analytical column with an 8- $\mu \mathrm{m}$ emitter (New Objective, Woburn, MA) packed to $25 \mathrm{~cm}$ with ReproSil-Pur C18-AQ, $1.9 \mu \mathrm{M}$ material (Dr. Maish $\mathrm{GmbH}$ ). Mobile phase A consisted of $2 \%$ acetonitrile; $0.1 \%$ formic acid and mobile phase B consisted of $90 \%$ acetonitrile; $0.1 \%$ formic acid. Peptides were then separated using the following steps: at a flow rate of $200 \mathrm{nl} / \mathrm{min}: 2 \% \mathrm{~B}$ to $6 \% \mathrm{~B}$ over $1 \mathrm{~min}, 6 \% \mathrm{~B}$ to $30 \% \mathrm{~B}$ over $84 \mathrm{~min}, 30 \% \mathrm{~B}$ to $60 \%$ B over $9 \mathrm{~min}, 60 \% \mathrm{~B}$ to $90 \% \mathrm{~B}$ over $1 \mathrm{~min}$, held at $90 \%$ B for $5 \mathrm{~min}, 90 \% \mathrm{~B}$ to $50 \%$ B over $1 \mathrm{~min}$ and then flow rate was increased to $500 \mu \mathrm{l} / \mathrm{min}$ as $50 \% \mathrm{~B}$ was held for $9 \mathrm{~min}$. Eluted peptides were directly electrosprayed into the MS with the application of a distal $2.3 \mathrm{kV}$ spray voltage and a capillary temperature of $300^{\circ} \mathrm{C}$. Full-scan mass spectra (Res = 60,$000 ; 400-1600 \mathrm{~m} / \mathrm{z}$ ) were followed by MS/MS using the "Top Speed" method for selection. High-energy collisional dissociation (HCD) was used with the normalized collision energy set to 35 for fragmentation, the isolation width set to 1.2 and a duration of $15 \mathrm{~s}$ was set for the dynamic exclusion with an exclusion mass width of $10 \mathrm{ppm}$. We used monoisotopic precursor selection for charge states $2+$ and greater, and all data were acquired in profile mode.

Database searching. Peaklist files were generated by Proteome Discoverer version 2.2.0.388 (Thermo Scientific). Protein identification was carried out using both Sequest $\mathrm{HT}^{76}$ and Mascot $2.5^{77}$ against the UniProt mouse reference proteome (57,220 sequences; 26,386,881 residues). Carbamidomethylation of cysteine, iTRAQ4plex (K), and iTRAQ4plex (N-term) were set as fixed modifications, methionine oxidation, and deamidation $(\mathrm{NQ})$ were set as variable modifications. Lys-C was used as a cleavage enzyme with one missed cleavage allowed. Mass tolerance was set at $20 \mathrm{ppm}$ for intact peptide mass and $0.3 \mathrm{Da}$ for fragment ions. 
Search results were rescored to give a final $1 \%$ FDR using a randomized version of the same Uniprot mouse database, with two peptide sequence matches (PSMs) required. iTRAQ ratio calculations were performed using Unique and Razor peptide categories in Proteome Discoverer.

RNA immunoprecipitation (RIP). RIP was performed following the Abcam protocol with minor modifications. 4T1 cells in culture were trypsinized and harvested, total 40 million cells were washed once with cold PBS before RIP experiment, then the cells were resuspended in $8 \mathrm{ml}$ PBS, $8 \mathrm{ml}$ nuclear isolation buffer $\left(1.28 \mathrm{M}\right.$ sucrose, $40 \mathrm{mM}$ Tris- $\mathrm{HCl} \mathrm{pH} \mathrm{7.5,} 20 \mathrm{mM} \mathrm{MgCl}_{2}$, and $4 \%$ Triton X100 supplemented with $100 \mathrm{U} / \mathrm{ml}$ SUPERase-IN and $1 \times$ Roche protease inhibitor cocktail), and $24 \mathrm{ml}$ nuclease-free water on ice for $20 \mathrm{~min}$ with frequent mixing. The cleared lysates were pelleted by centrifugation at $2500 \times g$ for $15 \mathrm{~min}$. Pellets resuspended in $4 \mathrm{ml}$ RIP buffer $(150 \mathrm{mM} \mathrm{KCl}, 25 \mathrm{mM}$ Tris pH 7.4, $5 \mathrm{mM}$ EDTA, $0.5 \mathrm{mM}$ DTT, and $0.5 \% \mathrm{NP}-40$ substitute supplemented with $100 \mathrm{U} / \mathrm{ml}$ SUPERaseIN and 1X Roche protease inhibitor cocktail) and sonicated for 5 min using BioRuptor Pico water bath sonicator $\left(30 \mathrm{~s}\right.$ ON/OFF) at $4{ }^{\circ} \mathrm{C}$. The lysates were cleaned by centrifugation at $13,000 \times g$ for $10 \mathrm{~min}$. The supernatant was collected and separated, then incubated with $4 \mu \mathrm{g}$ PURB antibody (Proteintech, 18128-1AP), PURA antibody (Abcam, ab125200), or rabbit isotype IgG control for $2 \mathrm{~h}$ to overnight at $4{ }^{\circ} \mathrm{C}$ with gentle rotation. In all, $80 \mu \mathrm{l}$ of protein A beads (Thermo Fisher, 88845) for rabbit antibody were added into the reactions and incubate for $1 \mathrm{~h}$ at $4{ }^{\circ} \mathrm{C}$ with gentle rotation. After washing three times with RIP buffer and once with PBS, beads were collected for immunoblot analysis and RNA extraction for qRT-PCR. Primers for RIP qRT-PCR can be found in Supplementary Data 8.

Western blot analysis. SDS Polyacrylamide gel electrophoresis (SDS-PAGE): Boiled samples were loaded to $10 \%$ a home casting polyacrylamide gel or MiniPROTEAN TGX Precast Protein Gels (BIO-RAD) for protein separation. Transfer: Proteins were transferred from the gel to 0.2- $\mathrm{m}$ PVDF membrane (the PVDF membrane was pre-activated using $100 \%$ methanol) using a wet transfer method. For most target proteins (MW between 14 and $116 \mathrm{kDa}$ ), the transfer setting was $100 \mathrm{~V}$ for 70 min using $1 \times$ Tris-glycine transfer buffer supplemented with $20 \%$ methanol; for tensin 1 , the transfer setting was $30 \mathrm{~V}$ overnight using $1 \times$ TrisGlycine transfer buffer supplemented with $10 \%$ methanol and $0.01 \%$ SDS. Blocking: Before the process, the membrane was stained with Ponceau $S$ for 1 min to check the transferred pattern, and de-stained completely with $1 \times$ PBS with $0.1 \%$ Tween 20 (PBST). Then the membrane/blot was placed in blocking buffer (PBST with $5 \%$ nonfat milk) on an orbital shaker at room temperature for $1 \mathrm{~h}$ for blocking. Primary antibody incubation: Before the incubation, the blot was rinsed with PBST once. The blot was incubated in the primary antibody diluted in PBST on the orbital shaker at $4{ }^{\circ} \mathrm{C}$ overnight. The primary antibody dilution used in this study: 1:1000-1500 for Tensin1 antibody, 1:200 for PURA, 1:200 for PURB, and 1:5000-10,000 for actin antibody. Second antibody incubation: The blot was extensively washed with PBST three times for $10 \mathrm{~min}$ each on the orbital shaker with gentle agitation. After washing, the blot was placed in the secondary antibody diluted in PBST at room temperature for $1 \mathrm{~h}$ The HRP-conjugated secondary antibody dilution: 1:3000 for goat-anti-rabbit secondary antibody targeting tensin 1 antibody, and 1:10,000-20,000 for goat-anti-mouse secondary antibody targeting actin antibody. ECL detection: The blot was extensively washed with PBST three times for 10 min each on the orbital shaker with gentle agitation. After washing, the blot was incubated with Pierce ${ }^{m}$ ECL Western Blotting Substrate (Thermo Scientific, PI32209) at room temperature for 1 min with gentle agitation $\left(^{*}\right.$ light sensitive). The signal on the blot was detected by using X-ray film. Antibodies used in this study are provided in Supplementary Data 5.

\section{Chromatin immunoprecipitation (ChIP) coupled with quantitative PCR (ChIP-} qPCR). For Chromatin immunoprecipitation (ChIP), we followed a published protocol ${ }^{78}$. Cross-linking: 30 million 4T1 cells were harvested and cross-linked in $1 \%$ formaldehyde at room temperature for $20 \mathrm{~min}$, then the reaction was quenched using $0.125 \mathrm{M}$ glycine (Thermo Fisher, AAJ16405A7) at room temperature for 10 min. Samples were centrifuged at $2000 \times g$ for $5 \mathrm{~min}$ at $4{ }^{\circ} \mathrm{C}$, and cell pellets were collected. Sonication and immunoprecipitation (IP): Cell pellets were incubated with cell lysis buffer $(10 \mathrm{mM}$ Tris $\mathrm{pH} 8.0,10 \mathrm{mM} \mathrm{NaCl}, 0.2 \% \mathrm{NP}-40$ substitute) for $15 \mathrm{~min}$ on ice. The cell lysates were quickly centrifuged at $2500 \times \mathrm{g}$ for $30 \mathrm{~s}$ at $4{ }^{\circ} \mathrm{C}$, and the nuclei pellets resuspended in $1.5 \mathrm{ml}$ of nuclei lysis buffer $(50 \mathrm{mM}$ Tris $\mathrm{pH}$ 8.0, $10 \mathrm{mM}$ EDTA, $1 \%$ SDS) and incubated for $10 \mathrm{~min}$ on ice. Next, the samples were sonicated for $15 \mathrm{~min}$ using a BioRuptor Pico water bath sonicator (30 s ON/ OFF) at $4^{\circ} \mathrm{C}$. The sonicated samples were centrifuged at max speed for $15 \mathrm{~min}$ at $4{ }^{\circ} \mathrm{C}$, and supernatants were transferred into a new $1.5-\mathrm{ml}$ tube as sonicated chromatin samples. Before IP, $5 \%$ of the sample was transferred into a $1.5-\mathrm{ml}$ tube as Protein INPUT, and 5\% of the sample was transferred into a $1.5-\mathrm{ml}$ tube as DNA INPUT. For one IP, $1.5 \mathrm{ml}$ of sonicated chromatin from 30 million cells were diluted with $21 \mathrm{ml}$ IP-Dilution buffer ( $20 \mathrm{mM}$ Tris pH 8.0, $2 \mathrm{mM}$ EDTA, $150 \mathrm{mM}$ $\mathrm{NaCl}, 1 \%$ Triton X-100, $0.01 \%$ SDS,) and incubated with $4 \mu \mathrm{g}$ of PURB antibody or rabbit isotype IgG control. The sample tubes were incubated for $3 \mathrm{~h} 4^{\circ} \mathrm{C}$ with rotation, and $80 \mu$ of protein $\mathrm{A}$ beads for rabbit antibody was added into the sample tubes. Next, the samples were incubated at $4{ }^{\circ} \mathrm{C}$ overnight with rotation. Washing: After incubation, beads were separated and collected using a DynaMag ${ }^{\mathrm{m}}$ -
2 Magnet stand. Beads were washed once with IP-wash 1 buffer $(20 \mathrm{mM}$ Tris $\mathrm{pH}$ 8.0, 2 mM EDTA, $50 \mathrm{mM} \mathrm{NaCl}, 1 \%$ Triton X-100, $0.1 \%$ SDS), twice with high-salt buffer (20 mM Tris pH 8.0, $2 \mathrm{mM}$ EDTA, $500 \mathrm{mM} \mathrm{NaCl}, 1 \%$ Triton X-100, $0.01 \%$ SDS), once with IP-wash 2 buffer (10 mM Tris pH 8.0, 1 mM EDTA $0.25 \mathrm{M} \mathrm{LiCl}$, $1 \%$ NP-40 substitute, $1 \%$ sodium deoxycholate), twice with TE buffer (10 mM Tris$\mathrm{Cl}, 1 \mathrm{mM}$ EDTA, $\mathrm{pH}$ 8.0). Elution and reverse cross-linking: Bead-bound chromatin was eluted in $800 \mu \mathrm{l}$ nuclear lysis buffer by heating at $65^{\circ} \mathrm{C}$ for $15 \mathrm{~min} .48 \mu \mathrm{l}$ of $5 \mathrm{M} \mathrm{NaCl}$ was added to the $800 \mu \mathrm{l}$ of eluted chromatin, followed by incubation at $65^{\circ} \mathrm{C}$ overnight for reverse cross-linking. DNA purification and analysis: After reverse cross-linking, DNA was treated with RNaseA (Sigma-Aldrich, R4642) and proteinase K (Viagen Biotech, 501-pk), followed by purification using QIAGEN PCR purification kit (QIAGEN, 28104). GPCR was performed on a ABI QuantStudio 6 Flex Real-Time PCR System. ChIP-qPCR primers can be found in Supplementary Data 8.

Single-molecule RNA fluorescence in situ hybridization (FISH). For singlemolecule RNA-FISH, custom Type-6 primary probes targeting MaTAR25, LINC01271, and other IncRNAs were designed and synthesized by Affymetrix. For RNA-FISH on cultured cell samples, Affymetrix View ISH Cell Assay Kit (Thermo Fisher, QVT0050) reagents were used. Cultured cells were harvested and seeded onto acid-cleaned \#1.5 glass coverslips (Electron Microscopy Sciences, 72230-01) for 24 -h incubation to $70 \%$ confluence, cell samples then were fixed in freshly prepared 4\% paraformaldehyde (PFA) (Electron Microscopy Sciences, 19200). Cells were then permeabilized and protease digested before hybridization. For RNA-FISH on formalin-fixed paraffin-embedded (FFPE) tissue sections of breast tumors and metastases, Affymetrix ViewRNA ISH Tissue 1-Plex Assay kit (Thermo Fisher, QVC0001) reagents were applied. Sections on slides were deparaffinized, protease digested and fixed with $10 \%$ NBF before hybridization. QuantiGene ViewRNA probe hybridizations were performed at $40^{\circ} \mathrm{C}$ for $3 \mathrm{~h}$. The hybridization and signal amplification steps were performed according to the manufacturer's instruction, and nuclei were counterstained with DAPI (Thermo Fisher, D1306). Coverslips and tissue sections were mounted in ProLong Gold Antifade mounting medium (Thermo Fisher, P36930) before detection. Imaging was performed on Zeiss LSM 710/780 Confocal Microscope systems. Assays and resources used in this study are provided in Supplementary Data 7.

DNA FISH. Different mouse BAC clones (RPCI-23) were used as template included (MaTAR25), and (Tensin1). In all, $1 \mu \mathrm{g}$ of BAC DNA was used as a template for random priming reaction to generate amine-modified DNA, and amine-modified DNA was labeled with a reactive fluorescent dye as fluorescent probes according to the protocol provided with ARES ${ }^{\mathrm{mi}}$ Alexa Fluor ${ }^{\mathrm{mm}}$ DNA Labeling Kit (Thermo Fisher A21665 and A21669). For DNA FISH, we followed protocols ${ }^{79}$. Briefly, cultured cells were seeded onto $22-\mathrm{mm}^{2}$ glass coverslips (Corning) or etched grid coverslips (Bellco, 1916-92525), and incubated to $70 \%$ confluence in the $5 \% \mathrm{CO}_{2}$ incubator at $37^{\circ} \mathrm{C}$ Coverslips were fixed with freshly prepared $4 \%$ PFA for $20 \mathrm{~min}$ at room temperature, and permeablized in $0.5 \%$ Triton X-100/1X PBS for $5 \mathrm{~min}$ on ice. Probes were prepared for hybridization by mixing $2 \mu$ of probe with $5 \mu$ each of sheared salmon sperm DNA (Thermo Fisher, AM9680), mouse Cot1 DNA (Thermo Fisher, 18440016), and yeast tRNA (Sigma-Aldrich, R5636), dehydrating the probe mixture in the speed-vac, and then resuspending the probe in $10 \mu \mathrm{l}$ of deionized formamide (Thermo Fisher, AM9342). Just prior to hybridization, the probes were denatured at $95^{\circ} \mathrm{C}$ for $10 \mathrm{v}$, transferred to ice for $5 \mathrm{v}$, and then mixed with $10 \mu \mathrm{l}$ of $2 \times$ hybridization buffer ( $4 \times$ SSC, $20 \%$ dextran sulfate) and pipetted onto slides so that coverslips could be placed cell-side-down on the probe mixture for the hybridization reaction. Coverslips were sealed with rubber cement, and hybridization slides were placed in a humid chamber and incubating at $37^{\circ} \mathrm{C}$ overnight. Post-hybridization washing: Before washing, prepare $37^{\circ} \mathrm{C} 50 \%$ deionized formamide $/ 2 \times \mathrm{SSC}(\mathrm{pH} 7.0)$ washing solution 1 in a glass jar, and prepare $37^{\circ} \mathrm{C} 10 \mathrm{ml} 2 \times$ SSC and $37^{\circ} \mathrm{C} 10 \mathrm{ml} 1 \times$ SSC as washing solution 2 and 3 . Coverslips were removed from the hybridization slides and transferred to the glass jar containing washing buffer 1, and coverslips were incubated in a $37^{\circ} \mathrm{C}$ water bath for $30 \mathrm{~min}$ with gentle shaking. Coverslips were removed from washing solution 1 and put into $2 \times$ SSC for washing at $37^{\circ} \mathrm{C}$ for $15 \mathrm{v}$. Next, coverslips were placed into $1 \times \operatorname{SSC}$ for washing at $37^{\circ} \mathrm{C}$ for $15 \mathrm{~min}$, equilibrated in $4 \times$ SSC at room temperature for $5 \mathrm{~min}$. After these washing steps, nuclei were counterstained with DAPI. Coverslips were mounted in ProLong Gold Antifade mounting medium before detection. Coverslips were imaged using Zeiss LSM 710/780 Confocal Microscope systems.

Immunofluorescence (IF). For IF, we followed the protocol ${ }^{80}$ with minor modifications depend on applied antibodies. Cultured cells were harvested and seeded onto acid-cleaned \#1.5 glass coverslips for 24 -h incubation to $70 \%$ confluence, cell samples then were fixed in $4 \%$ formaldehyde for $20 \mathrm{~min}$. Samples were permeabilized in $0.2 \%$ Triton X-100 plus $1 \%$ bovine serum albumin (BSA) (Sigma-Aldrich, A2153) in PBS for 5 min on ice. After incubated in 1\% BSA in PBS for 30-min blocking, samples were incubated in the appropriate concentration (1:100 for paxillin, 1:100 for vinculin) of primary antibody for $1-2 \mathrm{~h}$ at room temperature, and incubated in diluted (1:200-1:400) secondary antibody solution (Alexa 594 conjugated) (Invitrogen, A-11012) with Phalloidin-iFluor 488 Reagent (stock solution:1000x; Abcam, ab176753) for $1 \mathrm{~h}$ in a humidified chamber at room 
temperature. After several washes, nuclei were counterstained with DAPI. Coverslips were mounted in ProLong Gold Antifade mounting medium before detection. Coverslips were imaged on the Zeiss LSM 710/780 Confocal Microscope systems.

Transmission electron microscopy (TEM). Cultured cells were harvested and seeded onto $10-\mathrm{cm}$ culture dishes for $24 \mathrm{~h}$, and fixed with $2.5 \%$ glutaraldehyde (EM grade) in $0.1 \mathrm{M}$ phosphate buffer $\mathrm{pH} 7.4$ at room temperature for $1 \mathrm{~h}$. The fixed cells were collected by using a cell scraper, washed several times in $0.1 \mathrm{M}$ phosphate buffer, and post fixed in $1 \%$ osmium tetroxide. Samples were dehydrated in ethanol (30\%, 50\%, 70\%, 80\%, 95\%, and 100\%), embedding in EMbed 812 Resin (Electron Microscopy Sciences, 14120) and polymerized. In all, 70-90-nm sections were cut on a Reichert-Jung ultramicrotome using a diamond knife (DiATOME). Sections were collected on copper grids, stained with UranyLess and lead citrate, and imaged with a Hitachi H-7000 TEM.

In vivo mouse model ASO injection. Three-month-old MMTV-Neu-NDL mice were divided into three cohorts (7-12 mice each), and each mouse in the cohort received either scASO or MaTAR25-specific ASO1 or ASO2 via subcutaneous injection $50 \mathrm{mg} / \mathrm{kg} /$ day twice per week. The injections were carried out for a period of 7 weeks, upon which at least one tumor from most of the control mice reached $2 \mathrm{~cm}$ in size. During the course of treatment, tumors were measured twice per week. At the end of the treatment period, the animals were euthanized and the primary tumors, lungs, livers, spleens, and intestines were collected. Collected lungs were fixed in $4 \%$ paraformaldehyde and incubated in $20-30 \%$ sucrose solution overnight, then frozen in OCT solution (Tissue-Tek, 62550-12). The lung OCT blocks were cross-sectioned 2-mm apart, and the lung sections were embedded horizontally to obtain serial sections of the entire lung. Other tissues were cut into two pieces. One part of each issue was snap-frozen using liquid nitrogen for further RNA extraction. The remaining tissues were fixed in $4 \%$ paraformaldehyde and paraffin-embedded, then formalin-fixed, paraffin-embedded (FFPE) tissue blocks were sectioned. All sections were stained with $\mathrm{H} \& \mathrm{E}$ following standard protocol, and slides were scanned and analyzed using an Aperio ImageScope pathology slide viewing system. All samples were processed and stained at the Cold Spring Harbor Laboratory Histology Shared Resource. Slides were scanned and examined using Aperio ImageScope software.

In vivo 4T1 cells injection. In all, 5-6-week-old female BALB/c mice and $4 \mathrm{~T} 1$ cells (control and MaTAR25 KO cells) expressing luciferase were used for 4T1 mammary fat pad and tail-vein injection experiments. For mammary fat pad injection, $1 \times 10^{5} 4 \mathrm{~T} 1$ control or MaTAR25 KO cells were injected orthotopically into the mammary fat pad of female BALB/c mice. Mice were monitored and primary tumors were measured every week. Mice were sacrificed, and tumors were collected at day 28 to compare the tumor growth rate between $4 \mathrm{~T} 1$ control groups and MaTAR25 KO groups.

For tail-vein injection, female BALB/c mice were injected intravenously with $1 \times 10^{5} 4 \mathrm{~T} 1$ control or MaTAR25 KO cells in the tail vein. Mice were monitored every week and sacrificed at day 21 . The mouse lungs were collected and imaged, and lung metastatic nodules were counted to compare the metastatic ability between $4 \mathrm{~T} 1$ control groups and MaTAR25 KO groups.

Quantification and statistical analysis. Statistics tests were performed and analyzed using Microsoft Excel and GraphPad Prism 7.0. $P$ value was calculated by paired Student's $t$ test, two-tailed. Significance was defined as $P<0.05$. $P$ values of results are provided in the Source Data file.

Outline of tools used in this study. UCSC genome browser (https://genome.ucsc. $\mathrm{edu} /)^{81}$

ensembl genome browser (http://useast.ensembl.org/index.html)

FANTOM5 (https://fantom.gsc.riken.jp/5/)

CPAT (http://lilab.research.bcm.edu/cpat/) 82

CPC (http://cpc.gao-lab.org/) 83

KM plotter (https://kmplot.com/analysis/)

TANRIC (https://ibl.mdanderson.org/tanric/_design/basic/query.html).

Reporting summary. Further information on research design is available in the Nature Research Reporting Summary linked to this article.

\section{Data availability}

The accession number for the RNA-seq and ChIRP-seq data reported in this study is GEO: GSE142169. The dataset identifier for the mass spectrometry proteomics data deposited to the ProteomeXchange Consortium via the PRIDE partner repository is: PXD017398. All data are available from the corresponding author upon reasonable request. Source data are provided with this paper.

Received: 27 January 2020; Accepted: 20 November 2020; Published online: 22 December 2020

\section{References}

1. Siegel, R. L., Miller, K. D. \& Jemal, A. Cancer statistics, 2020. CA Cancer J. Clin. 70, 7-30 (2020).

2. Li, C. I., Uribe, D. J. \& Daling, J. R. Clinical characteristics of different histologic types of breast cancer. Br. J. Cancer 93, 1046-1052 (2005)

3. Malhotra, G. K., Zhao, X., Band, H. \& Band, V. Histological, molecular and functional subtypes of breast cancers. Cancer Biol. Ther. 10, 955-960 (2010).

4. Curtis, C. et al. The genomic and transcriptomic architecture of 2,000 breast tumours reveals novel subgroups. Nature 486, 346-352 (2012).

5. Perou, C. M. et al. Molecular portraits of human breast tumours. Nature 406, 747-752 (2000).

6. Wallden, B. et al. Development and verification of the PAM50-based Prosigna breast cancer gene signature assay. BMC Med. Genomics 8, 54 (2015).

7. Eroles, P., Bosch, A., Perez-Fidalgo, J. A. \& Lluch, A. Molecular biology in breast cancer: intrinsic subtypes and signaling pathways. Cancer Treat. Rev. 38, 698-707 (2012).

8. Derrien, T. et al. The GENCODE v7 catalog of human long noncoding RNAs analysis of their gene structure, evolution, and expression. Genome Res. 22, 1775-1789 (2012).

9. Djebali, S. et al. Landscape of transcription in human cells. Nature 489, 101-108 (2012).

10. Guttman, M. et al. Chromatin signature reveals over a thousand highly conserved large non-coding RNAs in mammals. Nature 458, 223-227 (2009).

11. Wilusz, J. E., Sunwoo, H. \& Spector, D. L. Long noncoding RNAs: functional surprises from the RNA world. Genes Dev. 23, 1494-1504 (2009).

12. Bergmann, J. H. \& Spector, D. L. Long non-coding RNAs: modulators of nuclear structure and function. Curr. Opin. Cell Biol. 26, 10-18 (2014).

13. Ulitsky, I. \& Bartel, D. P. lincRNAs: genomics, evolution, and mechanisms. Cell 154, 26-46 (2013).

14. Mercer, T. R., Dinger, M. E., Sunkin, S. M., Mehler, M. F. \& Mattick, J. S. Specific expression of long noncoding RNAs in the mouse brain. Proc. Natl Acad. Sci. USA 105, 716-721 (2008)

15. Rinn, J. L. \& Chang, H. Y. Genome regulation by long noncoding RNAs. Annu. Rev. Biochem. 81, 145-166 (2012).

16. Amodio, N. et al. MALAT1: a druggable long non-coding RNA for targeted anti-cancer approaches. J. Hematol. Oncol. 11, 63 (2018).

17. Arun, G., Diermeier, S. D. \& Spector, D. L. Therapeutic targeting of long noncoding RNAs in cancer. Trends Mol. Med. 24, 257-277 (2018).

18. Fatica, A. \& Bozzoni, I. Long non-coding RNAs: new players in cell differentiation and development. Nat. Rev. Genet. 15, 7-21 (2014).

19. Morlando, M. \& Fatica, A. Alteration of epigenetic regulation by long noncoding RNAs in cancer. Int. J. Mol. Sci. 19, 570 (2018).

20. Sun, M., Nie, F. Q., Wang, Z. X. \& De, W. Involvement of lncRNA dysregulation in gastric cancer. Histol. Histopathol. 31, 33-39 (2016).

21. Tian, T. et al. The impact of lncRNA dysregulation on clinicopathology and survival of breast cancer: a systematic review and meta-analysis. Mol. Ther. Nucleic Acids 12, 359-369 (2018).

22. Zhang, H. et al. Long non-coding RNA: a new player in cancer. J. Hematol. Oncol. 6, 37 (2013)

23. Gupta, R. A. et al. Long non-coding RNA HOTAIR reprograms chromatin state to promote cancer metastasis. Nature 464, 1071-1076 (2010).

24. Godinho, M., Meijer, D., Setyono-Han, B., Dorssers, L. C. \& van Agthoven, T. Characterization of BCAR4, a novel oncogene causing endocrine resistance in human breast cancer cells. J. Cell. Physiol. 226, 1741-1749 (2011).

25. Xing, Z., Park, P. K., Lin, C. \& Yang, L. LncRNA BCAR4 wires up signaling transduction in breast cancer. RNA Biol. 12, 681-689 (2015).

26. Arun, G. et al. Differentiation of mammary tumors and reduction in metastasis upon Malat1 lncRNA loss. Genes Dev. 30, 34-51 (2016).

27. Arun, G. \& Spector, D. L. MALAT1 long non-coding RNA and breast cancer RNA Biol. 16, 860-863 (2019).

28. Tseng, Y. Y. et al. PVT1 dependence in cancer with MYC copy-number increase. Nature 512, 82-86 (2014).

29. Liu, B. et al. A cytoplasmic NF-kappaB interacting long noncoding RNA blocks IkappaB phosphorylation and suppresses breast cancer metastasis. Cancer Cell 27, 370-381 (2015).

30. Diermeier, S. D. et al. Mammary tumor-associated RNAs impact tumor cell proliferation, invasion, and migration. Cell Rep. 17, 261-274 (2016).

31. Petryszak, R. et al. Expression Atlas update-an integrated database of gene and protein expression in humans, animals and plants. Nucleic Acids Res. 44, D746-D752 (2016).

32. Murray, S. et al. TricycloDNA-modified oligo-2'-deoxyribonucleotides reduce scavenger receptor B1 mRNA in hepatic and extra-hepatic tissues-a comparative study of oligonucleotide length, design and chemistry. Nucleic Acids Res. 40, 6135-6143 (2012).

33. Seth, P. P. et al. Short antisense oligonucleotides with novel 2'-4' conformationaly restricted nucleoside analogues show improved potency without increased toxicity in animals. J. Med. Chem. 52, 10-13 (2009). 
34. Teplova, M. et al. Crystal structure and improved antisense properties of 2'-O(2-methoxyethyl)-RNA. Nat. Struct. Biol. 6, 535-539 (1999).

35. Chu, C., Qu, K., Zhong, F. L., Artandi, S. E. \& Chang, H. Y. Genomic maps of long noncoding RNA occupancy reveal principles of RNA-chromatin interactions. Mol. Cell 44, 667-678 (2011).

36. Chen, H., Ishii, A., Wong, W. K., Chen, L. B. \& Lo, S. H. Molecular characterization of human tensin. Biochem. J. 351, 403-411 (2000).

37. Hall, E. H., Daugherty, A. E., Choi, C. K., Horwitz, A. F. \& Brautigan, D. L. Tensin1 requires protein phosphatase-1alpha in addition to RhoGAP DLC-1 to control cell polarization, migration, and invasion. J. Biol. Chem. 284, 34713-34722 (2009).

38. Gyorffy, B. et al. An online survival analysis tool to rapidly assess the effect of 22,277 genes on breast cancer prognosis using microarray data of 1,809 patients. Breast Cancer Res. Treat. 123, 725-731 (2010).

39. Mouneimne, G. \& Brugge, J. S. Tensins: a new switch in cell migration. Dev. Cell 13, 317-319 (2007).

40. Parsons, J. T., Horwitz, A. R. \& Schwartz, M. A. Cell adhesion: integrating cytoskeletal dynamics and cellular tension. Nat. Rev. Mol. Cell Biol. 11, 633-643 (2010).

41. Balas, M. M. \& Johnson, A. M. Exploring the mechanisms behind long noncoding RNAs and cancer. Noncoding RNA Res. 3, 108-117 (2018).

42. Johnson, E. M., Daniel, D. C. \& Gordon, J. The pur protein family: genetic and structural features in development and disease. J. Cell Physiol. 228, 930-937 (2013).

43. Lasham, A., Lindridge, E., Rudert, F. \& Onrust, R. \& Watson. J. Regulation of the human fas promoter by YB-1, Pura and AP-1 transcription factors. Gene 252, 1-13 (2000).

44. Maamar, H., Cabili, M. N., Rinn, J. \& Raj, A. linc-HOXA1 is a noncoding RNA that represses Hoxal transcription in cis. Genes Dev. 27, 1260-1271 (2013).

45. Minn, A. J. et al. Genes that mediate breast cancer metastasis to lung. Nature 436, 518-524 (2005).

46. Li, J. et al. TANRIC: an interactive open platform to explore the function of IncRNAs in cancer. Cancer Res. 75, 3728-3737 (2015).

47. Bernau, K. et al. Tensin 1 is essential for myofibroblast differentiation and extracellular matrix formation. Am. J. Respir. Cell Mol. Biol. 56, 465-476 (2017).

48. Chen, H., Duncan, I. C., Bozorgchami, H. \& Lo, S. H. Tensin1 and a previously undocumented family member, tensin2, positively regulate cell migration. Proc. Natl Acad. Sci. USA 99, 733-738 (2002).

49. Saintigny, G., Bernard, F. X., Juchaux, F., Pedretti, N. \& Mahe, C. Reduced expression of the adhesion protein tensin 1 in cultured human dermal fibroblasts affects collagen gel contraction. Exp. Dermatol. 17, 788-789 (2008).

50. Okayama, A. et al. Identification of tyrosine-phosphorylated proteins upregulated during epithelial-mesenchymal transition induced with TGF-beta. J. Proteome Res. 14, 4127-4136 (2015).

51. Becker, C. et al. Regulation of IL-12 p40 promoter activity in primary human monocytes: roles of NF-kappaB, CCAAT/enhancer-binding protein beta, and PU.1 and identification of a novel repressor element (GA-12) that responds to IL-4 and prostaglandin E(2). J. Immunol. 167, 2608-2618 (2001).

52. Johnson, E. M. The Pur protein family: clues to function from recent studies on cancer and AIDS. Anticancer Res. 23, 2093-2100 (2003).

53. Kelm, R. J. Jr., Lamba, G. S., Levis, J. E. \& Holmes, C. E. Characterization of purine-rich element binding protein $\mathrm{B}$ as a novel biomarker in acute myelogenous leukemia prognostication. J. Cell Biochem. 119, 2073-2083 (2018).

54. Shi, J., Cheng, C., Ma, J., Liew, C. C. \& Geng, X. Gene expression signature for detection of gastric cancer in peripheral blood. Oncol. Lett. 15, 9802-9810 (2018).

55. Murugesan, S. N. et al. Expression and network analysis of YBX1 interactors for identification of new drug targets in lung adenocarcinoma. J. Genomics 6, 103-112 (2018).

56. Shibata, T. et al. Y-box binding protein YBX1 and its correlated genes as biomarkers for poor outcomes in patients with breast cancer. Oncotarget 9 , 37216-37228 (2018). 78.

57. Wang, L. G. et al. Androgen receptor overexpression in prostate cancer linked to Pur alpha loss from a novel repressor complex. Cancer Res. 68, 2678-2688 (2008).

58. Michailidou, K. et al. Association analysis identifies 65 new breast cancer risk loci. Nature 551, 92-94 (2017).

59. Gupta, G. P. \& Massague, J. Cancer metastasis: building a framework. Cell 127, 679-695 (2006).

60. Jin, X. \& Mu, P. Targeting breast cancer metastasis. Breast Cancer 9, 23-34 (2015).

61. Hua, Y. et al. Peripheral SMN restoration is essential for long-term rescue of a severe spinal muscular atrophy mouse model. Nature 478, 123-126 (2011).

62. Pandey, S. K. et al. Identification and characterization of modified antisense oligonucleotides targeting DMPK in mice and nonhuman primates for the treatment of myotonic dystrophy type 1. J. Pharmacol. Exp. Ther. 355, 329-340 (2015).
63. Wheeler, T. M. et al. Targeting nuclear RNA for in vivo correction of myotonic dystrophy. Nature 488, 111-115 (2012).

64. Bennett, C. F., Baker, B. F., Pham, N., Swayze, E. \& Geary, R. S. Pharmacology of antisense drugs. Annu. Rev. Pharmacol. Toxicol. 57, 81-105 (2017).

65. Hong, D. et al. AZD9150, a next-generation antisense oligonucleotide inhibitor of STAT3 with early evidence of clinical activity in lymphoma and lung cancer. Sci. Transl. Med. 7, 314ra185 (2015).

66. Sachs, N. et al. A living biobank of breast cancer organoids captures disease heterogeneity. Cell 172, 373-386 e310 (2018).

67. Conrad, T., Conrad, T. \& Ørom, U. A. Cellular fractionation and isolation of chromatin-associated RNA. Methods Mol. Biol. 1468, 1-9 (2017).

68. Langmead, B. \& Salzberg, S. L. Fast gapped-read alignment with Bowtie 2. Nat Methods 9, 357-359 (2012).

69. Heinz, S. et al. Simple combinations of lineage-determining transcription factors prime cis-regulatory elements required for macrophage and B cell identities. Mol. Cell 38, 576-589 (2010).

70. Dobin, A. et al. STAR: ultrafast universal RNA-seq aligner. Bioinformatics 29, 15-21 (2013).

71. Anders, S., Pyl, P. T. \& Huber, W. HTSeq-a Python framework to work with high-throughput sequencing data. Bioinformatics 31, 166-169 (2015).

72. Love, M. I., Huber, W. \& Anders, S. Moderated estimation of fold change and dispersion for RNA-seq data with DESeq2. Genome Biol. 15, 550 (2014).

73. Luo, W., Friedman, M. S., Shedden, K., Hankenson, K. D. \& Woolf, P. J. GAGE: generally applicable gene set enrichment for pathway analysis. $B M C$ Bioinforma. 10, 161 (2009).

74. Luo, W. \& Brouwer, C. Pathview: an R/Bioconductor package for pathwaybased data integration and visualization. Bioinformatics 29, 1830-1831 (2013).

75. Ross, P. L. et al. Multiplexed protein quantitation in Saccharomyces cerevisiae using amine-reactive isobaric tagging reagents. Mol. Cell Proteom. 3, 1154-1169 (2004)

76. Eng, J. K., McCormack, A. L. \& Yates, J. R. An approach to correlate tandem mass spectral data of peptides with amino acid sequences in a protein database. J. Am. Soc. Mass Spectrom. 5, 976-989 (1994).

77. Perkins, D. N., Pappin, D. J., Creasy, D. M. \& Cottrell, J. S. Probability-based protein identification by searching sequence databases using mass spectrometry data. Electrophoresis 20, 3551-3567 (1999).

78. Huang, Y. H. et al. POU2F3 is a master regulator of a tuft cell-like variant of small cell lung cancer. Genes Dev. 32, 915-928 (2018).

79. Hogan, M. S., Parfitt, D. E., Zepeda-Mendoza, C. J., Shen, M. M. \& Spector, D L. Transient pairing of homologous Oct4 alleles accompanies the onset of embryonic stem cell differentiation. Cell Stem Cell 16, 275-288 (2015).

80. Spector, D. L. \& Smith, H. C. Redistribution of U-snRNPs during mitosis. Exp. Cell Res. 163, 87-94 (1986).

81. Mudge, J. M. et al. Discovery of high-confidence human protein-coding genes and exons by whole-genome PhyloCSF helps elucidate 118 GWAS loci. Genome Res. 29, 2073-2087 (2019).

82. Wang, L. et al. CPAT: coding-potential assessment tool using an alignmentfree logistic regression model. Nucleic Acids Res. 41, e74 (2013).

83. Kong, L. et al. CPC: assess the protein-coding potential of transcripts using sequence features and support vector machine. Nucleic Acids Res. 35, W345-W349 (2007).

\section{Acknowledgements}

We thank members of the Spector lab for critical discussions and advice throughout the course of this study. We also acknowledge Dr. J. Erby Wilkinson (University of Michigan Medical School) for help with pathology and histology analysis. We thank Dr. William Muller (McGill University, Montreal) for providing MMTV-Neu-NDL mice, Dr. Mikala Egeblad (CSHL) for providing MMTV-PyMT mice, Dr. Fred R. Miller (Wayne State University) for providing 4T1 cells, Dr. Joan Massagué (Sloan Kettering Institute) for providing MDA-MB-231 LM2 cells, Dr. Bruce Stillman (CSHL) for providing the pET3a-Xenopus laevis histone H2B plasmid, and Dr. Scott Lyons (CSHL) for providing Efla-Luc2-mStrawberry plasmid. We would also like to thank the CSHL Cancer Center Shared Resources (Microscopy, Mass Spectrometry, Flow Cytometry, Animal, Histology, and Next-Gen Sequencing) for services and technical expertise (NCI 2P3OCA45508). This research was supported by NCI 5P01CA013106-Project 3 (D.S.), NCI P30CA008748 (E.B.), Susan G. Komen postdoctoral fellowship (S.D.) and NCI 1K99CA215362 (S.D.), CSHL/ Northwell Health (D.S.), and Manhasset Woman's Coalition Against Breast Cancer (S.B.).

\section{Author contributions}

K.C. and D.S. designed experiments, analyzed the data, and wrote the paper. K.C. performed most of the experiments. S.D. analyzed the RNA-seq, ChIRP-seq, and TCGA human BC patient data. A.Y. performed the cell cycle analysis, and assisted with CRISPR mediated KO and RNA antisense pulldown. L.B. performed the tissue RNA-FISH. S.B. performed the human organoid culture, organoid RNA extraction, and data analysis. S.R. assisted with the in vivo mouse model ASO injections and $4 \mathrm{~T} 1$ mammary fat pad injections. H.A. performed the TEM sample preparation and imaging. D.P. provided Mass spectrometry advice and 
data analysis. K.K. and T.B. provided human BC patient samples for organoid culture and FFPE slides. E.B. provided human BC patient FFPE slides. C.F.B. and F.R. designed and provided ASOs for in vitro and in vivo experiments. D.S. supervised the research.

\section{Competing interests}

D.L. Spector is a consultant to, and receives research support from, Ionis Pharmaceuticals. C.F.B. is the executive Vice President, Chief Scientific Officer at Ionis Pharmaceuticals. F.R. is the Vice President, drug discovery at Ionis Pharmaceuticals. The remaining authors declare no competing interests.

\section{Additional information}

Supplementary information is available for this paper at https://doi.org/10.1038/s41467020-20207-y.

Correspondence and requests for materials should be addressed to D.L.S.

Peer review information Nature Communications thanks the anonymous reviewer(s) for their contribution to the peer review of this work.
Reprints and permission information is available at http://www.nature.com/reprints

Publisher's note Springer Nature remains neutral with regard to jurisdictional claims in published maps and institutional affiliations.

\section{(c) (i)}

Open Access This article is licensed under a Creative Commons Attribution 4.0 International License, which permits use, sharing, adaptation, distribution and reproduction in any medium or format, as long as you give appropriate credit to the original author(s) and the source, provide a link to the Creative Commons license, and indicate if changes were made. The images or other third party material in this article are included in the article's Creative Commons license, unless indicated otherwise in a credit line to the material. If material is not included in the article's Creative Commons license and your intended use is not permitted by statutory regulation or exceeds the permitted use, you will need to obtain permission directly from the copyright holder. To view a copy of this license, visit http://creativecommons.org/licenses/by/4.0/.

(C) The Author(s) 2020 